\title{
Representative days selection for district energy system optimisation: a solar district heating system with seasonal storage
}

\author{
Bram van der Heijde ${ }^{\mathrm{a}, \mathrm{b}, \mathrm{c}, *}$, Annelies Vandermeulen ${ }^{\mathrm{a}, \mathrm{b}, \mathrm{c}}$, Robbe Salenbien $^{\mathrm{a}, \mathrm{c}}$, \\ Lieve Helsen ${ }^{\mathrm{a}, \mathrm{b}}$ \\ ${ }^{a}$ Energy Ville, Thor Park, Poort Genk 8310, 3600 Genk, Belgium \\ ${ }^{b}$ KU Leuven, Department of Mechanical Engineering, Celestijnenlaan 300 box 2421, 3001 \\ Leuven, Belgium \\ ${ }^{c}$ VITO, Boeretang 200, $2400 \mathrm{Mol}$, Belgium
}

\begin{abstract}
The design and operational optimisation of fourth generation district heating networks is a crucial step towards highly renewable energy systems of the future. In order to optimise such complex systems, a toolbox modesto (multi-objective district energy systems toolbox for optimisation) is being developed. Seasonal thermal energy storage is an essential technology to allow larger shares of renewable energy sources, yet large computational power is required for its representation in full-year operational optimisations, as a step towards district energy system optimal design. To decrease computational complexity, a technique with representative days able to include seasonal thermal energy storage systems is developed and validated. This methodology combines different part-solutions from literature, but also adds a novel aspect to safeguard the chronology of the optimisation problem. To validate the approach, the design optimisation of a fictitious solar district heating system with seasonal thermal energy storage is compared to different representative day optimisations in two cases. The operational optimisation is a linear optimisation problem, implemented using modesto; the design optimisation is built as a genetic algorithm, optimising the size of the storage and solar systems in the network. The validation exercise is done for the operational and for the design optimisation separately. This comparative
\end{abstract}

\footnotetext{
${ }^{*}$ Corresponding author. EnergyVille, Thor Park, Poort Genk 8310, 3600 Genk, Belgium E-mail address: Bram.vanderHeijde@energyville.be
} 


\section{Nomenclature \\ CHP Combined Heat and Power \\ DC Duration Curve \\ DES District Energy System \\ MILP Mixed Integer Linear Program \\ MIQP Mixed Integer Quadratic Pro- gram \\ OCP Optimal Control Problem}

RC Resistance Capacitance

RES Renewable Energy Source

SoC State of Charge

STC Solar Thermal Collector

STES Seasonal Thermal Energy Storage

TES Thermal Energy Storage

study shows that modelling with representative days adequately mimics the behaviour for the presented case. Furthermore, a solution speed-up in the order of 10-30 times is shown for the representative optimisations with respect to the full year optimisation, in line with the reduction of the number of variables.

Keywords: Thermal network, District heating, Optimal design, Seasonal thermal energy storage, Time aggregation

\section{Introduction}

In the transition to a sustainable and mainly renewable-based energy system, interest in energy storage in general, but more specifically thermal energy storage is increasing. There are two reasons for this gain in interest: firstly, the typical heat demand profile and the availability of solar thermal energy are largely out of phase, with almost no heat demand in Summer, but with the largest surplus of renewable energy in this season as well. Secondly, some renewable energy sources with large potential are not easily predicted or controlled, causing additional challenges to include them in energy systems. Both phenomena are a challenge to reconcile with security of supply requirements.

So in order to increase the renewable energy source (RES) potential in smart energy systems (see Lund et al. [1]), energy storage is needed. The addition of both storage units and RES requires high investments, and their control is often crucial for an adequate and cost-effective system operation. 
For optimal design of systems with seasonal thermal energy storage (STES) units, it is necessary to use a representation of at least a full year period in order to take seasonal phenomena into account. Furthermore, sufficiently accurate models with a minimum time resolution are needed for meaningful results.

An optimisation framework named modesto has been set up by Vandermeulen et al. 22, specifically targeted at this type of optimal design and control problems for district energy systems, currently with a focus on thermal energy systems. modesto is largely built around linearised optimisation models and allows to easily change network lay-outs and add or remove components. However, the linearity of the optimisation problem requires that only the operation of the system is variable and thus optimised, and the design parameters need to be fixed. The combination of both a long time duration and a high time resolution, needed for accurate modelling of STES systems, leads to excessively long calculation times. If we want to optimise the system design, multiple runs of modesto with varying design choices are needed. Because many optimisations are needed to explore the design parameter space, a speed-up of computation is essential.

\subsection{Design optimisation of district energy systems}

modesto is positioned in a framework of other similar optimisation studies for district energy systems (DES). While a comprehensive overview of studies optimising the integration of renewable energy sources and energy storage in district heating networks was presented by Olsthoorn et al. [3], a number of studies relevant to the subject of this paper is presented below. An optimisation approach for the topology of district energy systems was presented by Söderman and Pettersson [4], who presented an MILP (mixed integer linear program) for a DES including a thermal network and an electric grid, as well as thermal energy storage (TES) systems. The optimisation problem was limited to eight time instances, representing typical daily and nightly operation conditions for the four seasons. Weber [5] combined optimisation problems for design and control of poly-generation systems in district energy networks with different energy carriers, however without considering TES, again considering only limited temporal detail. Fazlollahi et al. [6] presented a multi-objective optimisation strategy for DES including district heating and poly-generation. The temporal complexity is managed by using 8 representative typical periods. They did not consider large-scale TES 
systems 1 An MILP integrating design, operation and network layout for an urban energy system with 12 buildings was presented by Morvaj et al. [7]. They considered 288 time steps, averaging the electric and heat load profiles for a whole year. Falke et al. [8] presented a similar multi-objective optimisation problem. They proposed a modular optimisation strategy, in which a rule-based control flow represented the operational layer. Furthermore, they included different levels of building renovation as a design choice, resulting in changed heat loads as an input to the optimisation algorithm. The variation of design choices was implemented as a genetic algorithm. The optimisation of the heating network layout was treated separately and used a heuristic algorithm.

Gabrielli et al. [9] compared a number of methods using representative days for integrated control and design optimisation in an MILP for a renewable energy system with a full year optimisation. Their optimisation framework includes two MILPs: one coarse optimisation with limited operational details for technology selection and sizing, followed by a more detailed optimisation of the operational aspects for the systems chosen by step 1 . Furthermore, they compared different time aggregation methods, one completely uncoupling the selected representative days, a second that explicitly couples seasonal variables across different representative days, and a final one without representative days, instead reducing the number of binary optimisation variables (namely the on/off variables). They found that the methods they proposed were in good agreement with the full resolution optimisation, with the distinction that the version with reduced binary variables was faster. Prina et al. [10] coupled an evolutionary design algorithm to the established software EnergyPlan to represent the operation of regional energy systems. They also accounted for the long-term investment planning problem, considering a changing energy mix over the years as obsolete technologies are replaced.

\subsection{Representative time slice methods considered in this study}

A recurring technique to speed up design optimisation in the aforementioned studies is to reduce the scale and complexity of the optimisation model by only considering a limited number of representative days or other time periods. Typically, these periods are chosen so as to represent the limits under

\footnotetext{
${ }^{1}$ Hence certainly no STES, but larger scale TES systems could also be used to act on shorter - daily or weekly - time scales.
} 
which the system must be able to operate - i.e., extreme days - but also the average behaviour of the system, such that the average of the representative set coincides with the annual average behaviour. In most of the studies presented above, the selection is based on heuristics (select one or two periods for each season, include days with extreme demand or production peaks).

Using representative days or time slices to represent a full year has furthermore been established in electrical energy system modelling. Nahmmacher et al. [11] present an overview of how representative time periods are selected to account for demand fluctuations. However, they argue that these methods are not yet sufficiently able to account for variability at the production side, i.e., with the introduction of uncontrollable RES. Their method is based on hierarchical clustering to select the most accurate representation of a full year.

Poncelet et al. [12] introduced a novel method to select representative time slices, namely an MILP-based selection of representative periods. The objective of the optimisation is to minimise the deviation between duration curves (DC) of the full year input profiles and their representation by means of representative time periods, where the number of selected periods, the number of times that these periods are repeated and starting day of each of the representative time periods can be varied. In a basic version, only the DC of the original input profiles is used, but additional DCs can be introduced to include data variability $\left.\right|^{2}$ or correlations between multiple input datasets. This method was compared against a number of other methods (random selection, hierarchic clustering and heuristic selection) and was shown to perform better when used in a reference optimisation problem.

This methodology of representative time slices was used in combined design and control optimisation of thermal systems by Patteeuw and Helsen [13]. They used the approach proposed by Poncelet to select six representative weeks, which is a compromise between accuracy and calculation time due to the complexity of the MILP optimisation problem. In their case, the full design optimisation was infeasible even on a supercomputer due to the size of the problem, so there is no information on how well the solution of the reduced design optimisation represents the actual optimum. However, it was shown that the DCs of the representative input profiles approximate those of the original data accurately. The focus of Patteeuw and Helsen [13] was

\footnotetext{
${ }^{2}$ I.e., the DCs of the one-step differences of the input profiles.
} 
on residential systems with hot water buffers, whose thermal capacity only allows for daily or weekly storage. Hence, the representative weeks can be modelled with periodic boundary constraints on the state of the heat-storing units. The study described in the current paper, however, considers seasonal energy storage effects and as a consequence these periodic boundary conditions cannot be applied, except for the one from the end to the beginning of the year.

Another example of thermal system optimisation with representative time slices is the work of Timmerman et al. [14]. While they used a different method of representative time slice selection than Poncelet et al. (namely, the method of Welsch et al. [15]), they did incorporate electrical and thermal energy storage systems, for which the duration of the load cycle is optimised. They presented an innovative method to account for the energy loss from these energy storage systems by using so-called discount factors. Timmerman et al. only described a simplified case to illustrate the effect of the use of representative time slices with an arbitrary time period selection. The results were not compared to a case with an equivalent full year optimisation.

Recently, Kotzur et al. [16] have proposed a method with which generic seasonal energy storage can be optimised both for control and design, and in which the representative days can be combined in any order. The order must be chosen such that the representative time series approach the original data as close as possible. Still, their results show a considerable difference in optimal design for an energy system with CHP, solar PV panels and battery storage, depending on the number of selected days as compared to the full year optimisation. They attribute the difference to a suboptimal choice and order of the representative days and suggest to combine an optimal selection of representative days be combined with their method.

A preliminary study (see van der Heijde et al. [17]) has already shown the suitability of the combination of the representative days approach for a very simple renewable energy hub, including a solar thermal collector array and a large TES tank, connected to a residential area. However, a large shortcoming identified in this study is the incorrect representation of the contribution of solar energy to the systems, often resulting in larger curtailment in representative optimisations than in the full optimisation problem, which will be studied further in this paper. 


\subsection{Other time aggregation methods}

Although a majority of studies uses representative time slices or typical periods, adapting the time step is also an option. For systems with large time constants (such as STES systems), it is possible to increase the time step without causing numerical instabilities. Most temporal discretisation schemes run into instabilities if the time step is increased too much, which is what prevents doing so in most cases. However, Renaldi and Friedrich [18] presented a methodology using multiple time grids for the operational optimisation of an energy system with solar thermal collectors, a gas boiler, short-term buffer storage tank and a borehole STES system. They concluded that the optimisation using a multiple-uniform time grid (large time step for STES, small time step for the other components) could reduce the calculation time by up to $94 \%$ with respect to the single-uniform time grid. However, as the focus of this paper is on representative days, the method of Renaldi and Friedrich will not be treated in the rest of the paper. Before Renaldi, Floros et al. [19] described the simulation of two smart-grid cases (a district cooling network and an energy market model), where they applied a quantised state solver (QSS). This solver method monitors the change in all model states, but only effectively changes the state if the increase or decrease exceeds a predefined "quantum". This implies that the number of state derivative evaluations becomes much lower than in a usual integration approach. Floros et al. concluded the QSS method performed much better compared to DASSL and Runge-Kutta solvers, reducing the simulation time by orders of magnitude while keeping the same magnitude of model error. The authors suggested this method could also be coupled to a dynamic optimisation of energy systems as a future recommendation. Note that, although Floros' study is not a design optimisation example, a simulation with a QSS solver could be used to implement the fitness evaluation in the operational level of a two-level optimal design approach.

\subsection{Contribution of this paper}

The literature review has established that a number of methods to reduce the complexity of optimisation problems for energy systems (and thereby reduce calculation time) already exists. These methods are complementary, but the combination of all aspects, in particular to a solar district heating case, is a clear gap in research.

This paper aims at presenting and validating a framework for the design optimisation of a district energy system with seasonal thermal energy storage, 
using an optimal selection of representative days to limit the complexity of the problem, without trading in accuracy. The combination of these three aspects (optimal selection of representative days, representative days combined with seasonal energy storage and design of a solar district energy system) was not encountered before. Apart from this novel combination of aspects, this paper furthermore presents a new method to preserve the chronology of the selected representative days in the optimisation algorithms, which is needed to correctly represent seasonal effects.

The first objective is to introduce an improved method to model seasonal energy storage with representative days into modesto. Secondly, we validate the results of the operational optimisation with representative days as compared to the full year operational optimisation. This is done by comparing the operational optimisation outcomes for different representative days selections with the same reference designs. Finally, we want to compare the optimal design results for different representative day selections and the full year case. Hence, for each selection of representative days as well as the full year case, a full genetic algorithm is run. The resulting design pareto fronts are checked for similarity.

The scope of this paper is limited to the presentation and validation of the proposed methodology. The outcome of the design algorithm will be discussed mostly in function of the verification of the presented methodology. The design results for a more realistic case study will be treated in detail in a separate paper.

\section{Method}

This section elaborates on the methods that were used to produce the results presented in this paper. Firstly, we explain how the representative days were selected and how their chronology was reintroduced afterwards, with a short discussion on how STES is modelled under representative time slices. Then, the optimisation models and the genetic algorithm that were used are described.

\subsection{Representative days}

This section describes the selection of representative days and the reconstruction of a chronological order with this selection. 


\subsubsection{Selection of representative days}

The selection of representative days is transformed into an MILP, as described by Poncelet et al. [12]. In this study, we make a selection based on the aggregated heat demand, output of the solar thermal collectors (STC) per collector unit area, outside temperature, electricity price and correlation between solar radiation and heat demand. The correlation is chosen to get a more realistic match between solar output and heat demand ${ }^{3}$, which is important for determining the chronology in the next step.

The optimisation problem minimises the difference of the DCs $4^{4}$ of the full year and the representative year for each of the time series. To this end, a set of representative days is selected from the year ${ }^{5}$, and for each representative day a weight is determined. This weight can be seen as the number of repetitions of each of the selected days to reconstruct a full year's worth of data. The sum of the weights should be equal to the number of days in the year (in this case). Since we are looking at actual repetitions to reconstruct a full year profile, we constrain the weights to be integers. Figure 1 provides a visual representation of how the representative days that are selected are repeated or weighted.

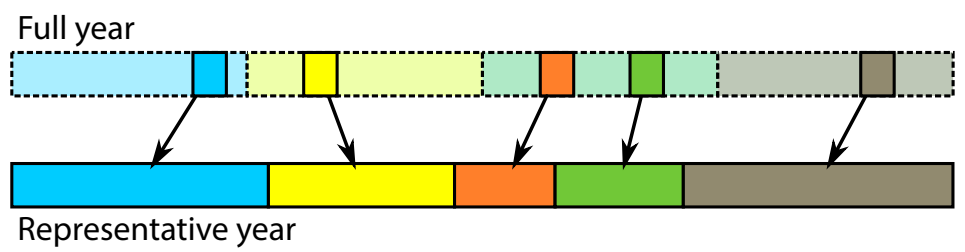

Figure 1: Schematic representation of selection of representative days and recombination to a full year duration. In this case, at least one day from each season is selected; the days are repeated for a well-chosen number of times, such that the representative year has the same number of days, but also such that the resulting DC resembles that of the original data.

A disadvantage inherent to time aggregation with representative days is that chronology is lost. The chronology is however essential for the opera-

\footnotetext{
${ }^{3}$ I.e., low heat demand when the solar radiation is high and vice versa.

${ }^{4} \mathrm{~A}$ duration curve (DC) is a function that maps the values of each of the profiles (e.g., heat demand, electricity cost...) onto a time duration, during which this value is exceeded in the full profile. This function is easily obtained by sorting the values of each of the time series in descending order.

${ }^{5}$ The number of representative days in the resulting selection is predefined by the user.
} 
tional optimisation of seasonal storage, and hence the next paragraph will describe how chronology can be restored. In Figure 1, this would mean that the representative year would not consist of 5 long blocks with the same colour, but that the repetitions of different selected days can form a sequence, such that not only the DC is approximated, but also the temporal behaviour.

\subsubsection{Chronological ordering}

After an optimal set of representative days has been chosen, they are reassigned to the days of the year such that every day in the data is represented by one of the representative days. The selection of which days are in the set

of representative days minimises the error with respect to the DCs, whereas the chronological ordering minimises the squared error of the representative data sets with regard to the original data sets.

The combination of the selection of the optimal days to represent the studied period, the number of times these days have to be repeated and the rearrangement for chronology is visualised for a simple example in Figure 2.

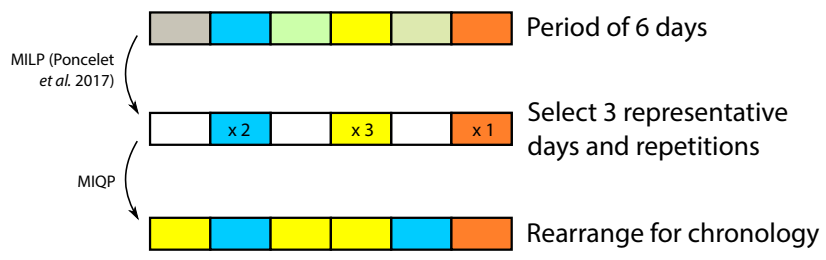

Figure 2: Example of a 'year' consisting of 6 different days. The year must be represented by 3 representative days. The first step is to select which days are optimal to represent the full data set and how many times they must be repeated to end up with the same total duration. After that, the MIQP orders the days in a new chronology that minimises the error with the original data sets.

This problem is formulated as an MIQP (mixed integer quadratic program): 


$$
\begin{array}{rlrl}
\min _{w} & \sum_{s \in S} \sum_{d \in D} \sum_{t \in T}\left(f_{s}(d, t)-g_{s}(d, t)\right)^{2} & \\
\text { s.t. } & & \\
\sum_{d \in D} w(d, r) & =n(r)-1 & & \\
w(d, r=d) & =1 & & \\
\sum_{r \in R} w(d, r) & =1 & & \\
\sum_{r \in R} w(d, r) \cdot f_{s}(r, t) & =g_{s}(d, t) & &
\end{array}
$$

where $d$ is the day of the year (in set $D=[0,364]$ ), $t$ is the timestep during the day (such that $t \in T=\left[0, n_{\text {steps }}-1\right]$ ), $r$ is one of the days in the set of selected representative days $R . w(d, r) \in\{0,1\}$ is the binary selection variable, taking value 1 if representative day $r$ is selected for day $d$. $s$ is the index of all time series in set $S$, with according value $f_{s}(d, t) .^{6}$ The reconstructed year has values $g_{s}(d, t) . n(r)$ denotes the weight or number of repetitions of representative day $r$.

(1c) makes sure that the representative days are selected to represent themselves in the recombined profile, e.g., that when day 3 is selected as a representative day, it is selected to represent day 3 in the full year profile. This implies that the number of repetitions of this representative day for the rest of the year is decreased by one, as indicated in constraint (1b). (1d) indicates that every day of the year has to be assigned exactly one representative day. Finally, (1e) assigns the time series data of the selected representative day to the reconstructed time series $g_{s}(d, t)$.

Furthermore, the contribution to the objective of each of the input profiles $s$ to be represented can be weighted. The weight is to be included in the objective function summation. This allows changing the relative importance of each of the input profiles.

The resulting annual profiles and DCs can be consulted in Appendix A.

\footnotetext{
${ }^{6}$ In this paper, the different time series $s$ are the heat demand profiles of the three neighbourhoods, the ambient temperature profile, the solar irradiation on a tilted unit surface and the electricity spot price. The correlation between solar radiation and the sum the of heat demands is taken in account only for the selection of the representative days.
} 


\subsection{Optimisation model}

This section describes the optimisation model that is tested with the time aggregation method from Section 2.1.

Note that the selection of the representative day set only depends on which input data time series are chosen for the design optimisation problem ${ }^{7}$ The representative day selection is not influenced by the considered design options or energy system configurations for the design optimisation. Hence, the representative days only have to be selected once before the start of the design algorithm.

\subsection{1. modesto}

A detailed description of modesto is provided by Vandermeulen et al. [2]. In short, modesto is a Python toolbox that interfaces with Pyomo [20] to construct control optimisation problems for district energy systems (DES). The goal is to easily define DES structures without having to program every single component from scratch. The toolbox has a library of linear models and optimises mass and heat flows in the system. In this work, modesto is used to construct the design problem as an optimal control problem (OCP) for a full year with perfect predictions of the boundary conditions.

Heat pumps are modelled as heat sources with a fixed coefficient of performance (COP), in this study 2.9. They translate the required heat injection to the network into an equivalent compressor energy use, which in its turn leads to an electricity cost using the variable electricity price.

The STC panels are modelled according to norm EN 12975-2 [21], namely using a temperature dependent absorption efficiency. The thermal output of the STC is modelled as:

$$
\dot{Q}_{\text {out }}(t)=A \cdot\left(\eta_{0} \dot{Q}_{\text {sol }}(t)-a_{1}\left(T_{m}-T_{a}(t)\right)-a_{2}\left(T_{m}-T_{a}(t)\right)^{2}\right)
$$

In this equation, $\dot{Q}_{\text {out }}$ represents the heat output, $\dot{Q}_{\text {sol }}$ is the solar irradiance on the unit surface and $A$ is the collector surface area. $\dot{Q}_{\text {sol }}$ is calculated beforehand assuming a south-oriented collector, tilted 40 deg with respect to the horizontal plane. The effect of the solar incidence angle on the panel on the transmission and reflection of the solar irradiance is neglected for simplicity.

\footnotetext{
${ }^{7}$ Input profiles can be the hourly heat demand, solar radiation, electricity price profiles, etc.
} 
$\eta_{0}, a_{1}$ and $a_{2}$ are efficiency parameters that are measured by the STC panel producers according to the European Standard $8 T_{a}$ is the ambient temperature, whereas $T_{m}$ represents the mean panel temperature. We assume $T_{m}$ to be the average of the supply and return temperature of the network, which would amount to a linear temperature increase along the collector pipe. When the heat output would become negative according to (2), it is set to be exactly $0 \mathrm{~W}$.

The district heating network is simulated with a nominal supply and return temperature that is matched with the high and low temperature from the TES systems in the network. Based on these nominal temperatures, the heat losses are calculated (see van der Heijde et al. [23]). However, an assumption of fixed heat losses is incompatible with the behaviour of the pipes at low mass flow rates 9 As a workaround, the heat losses increase linearly with the mass flow rate, such that the nominal heat loss is reached at the economically optimal mass flow rate through the pipe. In addition, also the energy required for circulating the water through the network is approximated using a set of linear inequality constraints that are based on the actual pumping power curve. This curve is a third-degree polynomial function of the mass flow rate.

The control optimisation problem in modesto also requires an objective function. Since existing networks will most likely be operated with the goal of minimising operative cost, this objective is chosen over an objective of minimal energy use or minimal $\mathrm{CO}_{2}$ emissions. Included in the operative cost objective function are the cost of electricity for the central heat pump and for the pumping power.

\subsubsection{Modelling seasonal storage with representative days}

The STES model is based on the model described by Vandewalle and D'haeseleer [24], using a perfectly stratified tank with fixed high and low temperatures. The same methodology is applicable to other storage models, as shown by Kotzur et al. [16]. The choice for this idealised storage model is based on the assumption of fixed supply and return temperatures of the

\footnotetext{
${ }^{8}$ In this paper, $\eta_{0}$ is $0.839, a_{1}$ is $2.46 \mathrm{~W} /\left(\mathrm{m}^{2} \mathrm{~K}\right)$ and $a_{2}$ is $0.0197 \mathrm{~W} /\left(\mathrm{m}^{2} \mathrm{~K}^{2}\right)$, corresponding to an Arcon Sunmark HT-SolarBoost 35/10 collector. These numbers were retrieved from the online SPF Collector Catalog [22]

${ }^{9}$ The fact that the heat loss cannot be larger than the heat input at one side of the pipe introduces a non-convex constraint in an otherwise linear optimisation program.
} 
THERNET. A perfectly mixed storage would have very similar heat losses (see Steen et al. [25]), but coupling it to a network with a fixed supply temperature without adding a system to upgrade the heat before injecting it into the network would mean a violation of the Second Law of thermodynamics. A more advanced model for a seasonal thermal storage pit is described by Sorknæs [26, but this model again assumes variable high and low temperatures in the model. This is left for further study.

In order to make the model compatible with interconnected representative days, the method of Kotzur et al. [16] is applied. The large reduction in optimisation variables is a result of the requirement that the heat and mass flows are the same in every repetition of the same representative day.

For the seasonal storage system (see Figure 3), this means that the relative increase ordecrease of the state of charge (SoC) will be also the same on every repetition of that same day.

The $\mathrm{SoC}$ is defined as:

$$
\mathrm{SoC}=\frac{Q_{\text {stor }}-Q_{\min }}{Q_{\max }-Q_{\min }},
$$

where $Q_{\max }$ and $Q_{\min }$ are the respective maximal and minimal energy storage levels of the STES system, and $Q_{\text {stor }}$ is the actual level. Because of the ideally stratified storage model, the energy storage level is determined by the position of the stratification surface, or equivalently by the share of the hot temperature volume compared to the total volume of the storage system. When all of the water in the storage tank is at the high temperature, $Q_{\max }$ is reached and the $\mathrm{SoC}$ is $100 \%$, and vice versa for $Q_{\min }$.

However, an additional variable that represents the base SoC for the whole day is introduced. The relative state change is required to start on a $\mathrm{SoC}$ of 0 (negative relative states are allowed), and the summation of the base state of charge and the relative change trajectory results in the effective state trajectory of the storage tank. As such, only one extra variable for the base SoC for each of the 365 days needs to be introduced. Heat losses are associated with both the base and relative $\mathrm{SoC}$ variables.

Finally, the storage states must be constrained for continuity and to stay within the minimal and maximal SoC. So firstly, the sum of the final relative $\mathrm{SoC}$ and the base state of charge for that day must be equal to the base SoC for the next day ${ }^{10}$. The final $\mathrm{SoC}$ on the last day of the year must also be

\footnotetext{
${ }^{10}$ Note that the relative SoC at the start of each day is defined as 0 and does not appear
} 

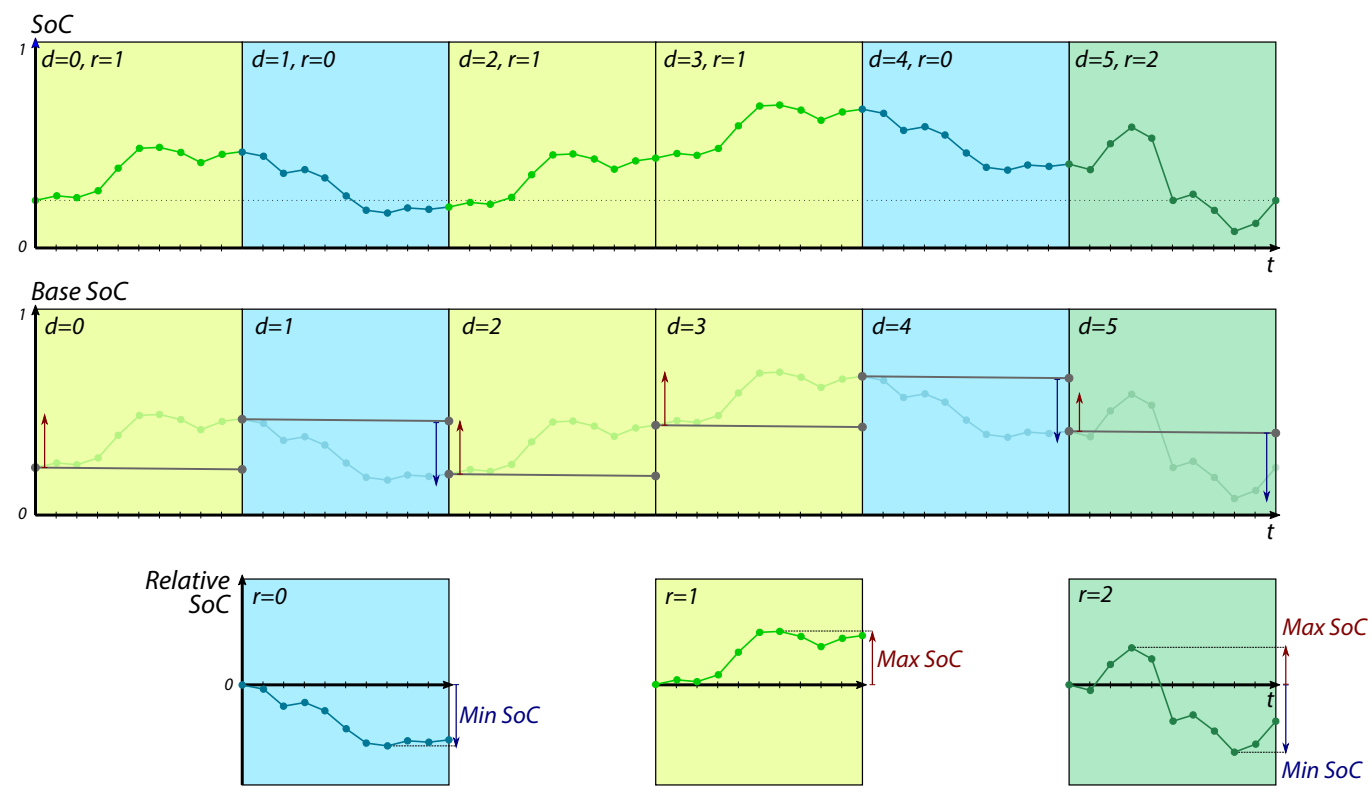

Figure 3: Visualisation of Kotzur's seasonal storage model. The example is a continuation of that in Figure 2. The top row shows the complete SoC evolution, the middle row shows the evolution of the daily base $\mathrm{SoC}$ and the bottom row shows the evolution of the relative SoC for each of the representative days.

The small red and blue arrows indicate the maximum (up, red) and minimum (down, blue) SoC for each of the illustrated representative days. Since representative days 0 and 1 have no maximal, respectively minimal SoC different from zero, hence no arrow is drawn. On the second row, the sum of the base SoC at the start of the day and the maximal relative SoC of the relevant representative day should not be larger than 1, whereas the sum of the base SoC at the end of that day and the (negative) minimal relative SoC should not be smaller than 0 . 
equal to the first SoC on the first day of the same year.

Secondly, two auxiliary variables are defined per representative day, containing the extreme points of the relative SoC trajectory for that day. The variables are constrained to be respectively larger and smaller than any of the relative SoC for each of the representative days. By adding these variables to the base SoC for every day in the year, the model checks whether the resulting extreme SoC remains within the bounds of the tank. The maximal relative $\mathrm{SoC}$ is added to the base $\mathrm{SoC}$ at the beginning of the day (the base SoC reduces slightly because of heat losses) and the minimal relative SoC is added to the base SoC at the end of the day. The reader is referred to Kotzur et al. [16] for all further details.

\subsubsection{Design optimisation}

The design optimisation algorithm is implemented as a genetic algorithm using the DEAP toolbox [27] in Python. The genetic algorithm is based on an NSGA-II selection operator [28] and uses a simulated binary crossover operator with a polynomial bounded mutation operator. In addition, a Hall of Fame with all pareto optimal solutions out of all previous individuals is kept. Whenever a new generation is started, the selection is made from the combination of this Hall of Fame and the individuals from the last generation. This avoids that optimal solutions are lost due to random variations over the generations.

Every individual in the genetic algorithm is an instance of modesto with a minimal cost objective. Hence, the optimal control for one year is computed with running cost minimisation as the aim. A flow chart illustrating the different steps in the genetic algorithm is shown in Figure 4 .

The design is evaluated for optimality based on two objectives, namely the annual (electrical energy) imported from outside the district energy system ${ }^{11}$ and the total annualised costs. The total annualised costs $c_{a}$ consist of the annual operation $\operatorname{costs} c_{o p, a}$ as calculated by modesto, increased by the annualised investment and fixed annual maintenance cost $c_{\text {maint }, a}$ :

$$
c_{a}=I_{a}+c_{o p, a}+c_{\text {maint }, a}
$$

\footnotetext{
in the sum

${ }^{11}$ I.e., the electrical energy used by the central heat pump at the Production node, and energy used by the pumps in the network to overcome the linearly approximated pressure losses
} 


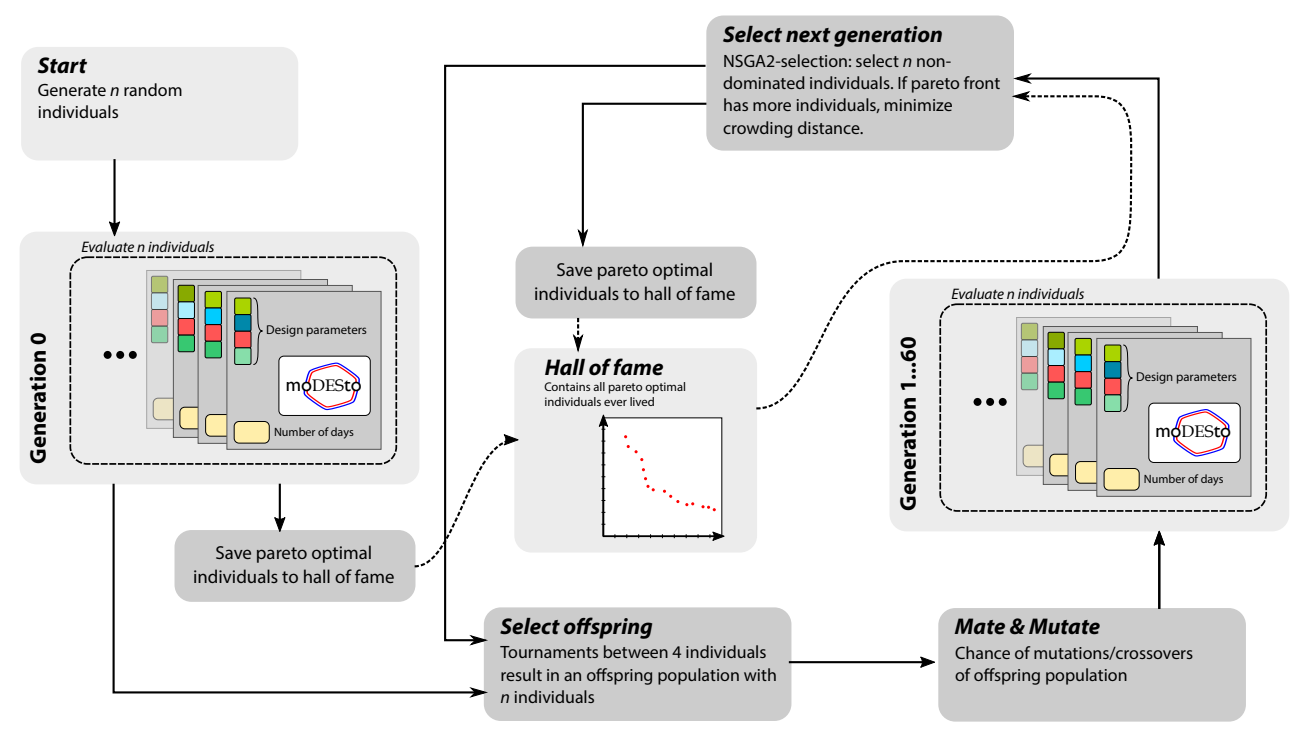

Figure 4: Flow chart illustrating the different steps in the genetic algorithm.

The annualised investment cost $I_{a}$ is calculated using the capital recovery factor 12 ;

$$
I_{a}=I_{t o t} \frac{(1+i)^{\tau} i}{(1+i)^{\tau}-1}
$$

where $I$ stands for the investment, with subscripts $a$ and tot denoting the annualised and total investment respectively. $\tau$ stands for the economic lifetime of the technology and $i$ is the interest rate, taken as $3 \%$ in this paper, assuming a public investment on a long term ${ }^{13}$

The annual fixed maintenance cost is a percentage of the total investment. The economic lifetime for the included technologies and the fixed maintenance fraction were taken from the EnergyPlan Cost Database [29]. All economic input data is summarised in Appendix B.

\footnotetext{
${ }^{12}$ The capital recovery factor assumes that at the end of its economic lifetime, each component is replaced by the same unit at the same cost. This neglects the fact that in the future, other technologies might be more interesting and that prices may change, but this is outside the scope of this paper.

${ }^{13}$ Given the +30 year economic lifetime of a district heating grid.
} 


\section{Reference case}

A fictitious reference case is set up for comparison of different representative days selections. A branched district heating network connects 3 neighbourhoods, namely Waterschei Garden City, Termien East and Termien West. These are residential neighbourhoods that actually exist in the Belgian city of Genk. A GIS database was used to formulate models of all residential buildings belonging to each of the neighbourhoods. These models were simulated using typical meteorological data for the Belgian climate ${ }^{14}$ and stochastic user behaviour to generate a heat demand profile for one year. For further information, the reader is guided to Appendix Cland to the work of De Jaeger et al. 31.

The lay-out of the reference neighbourhood is shown in Figure 5 .

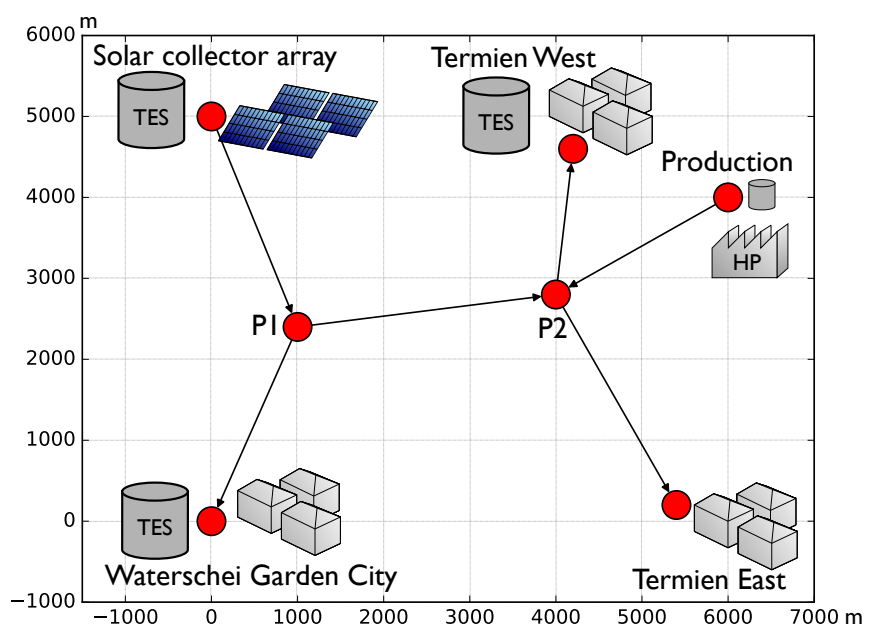

Figure 5: Lay-out of the reference case

At the production site, a large heat pump is installed with a buffer tank to bridge moments of peak demand. This tank has a limited volume of only $3000 \mathrm{~m}^{3}$, but uses the same model as the rest of the TES units. Its volume is

\footnotetext{
${ }^{14}$ This data set is included in the IDEAS Modelica Library, see Jorissen et al. [30. The data represents a typical meteorological year as measured at the weather station in Uccle, Belgium.
} 
fixed in the results discussed in Section 4.1, but is an optimisation decision variable in Section 4.2 .

A solar collector array (STC) is included at the indicated location, together with a STES unit. Two large STES units are installed at the neighbourhood nodes Waterschei and Termien West.

\section{Results}

\subsection{Validation of representative days for operational optimisation}

A first step is to check if the operational optimisation of the reference DES yields the same - or at least comparable - results for different sets of representative days and for the full year optimisation. To do this, the reference case of Section 3 is optimised for a number of deterministic variations of three design parameters in the network. The possible values of each of the design parameters and a description are listed in Table 1. All possible combinations lead to a total of 48 cases to be computed for each of the representative days selections and the full year operational optimisation.

This comparison is schematically represented in Figure6.

\subsubsection{Comparing the outcome of modesto}

The multitude of results of interest makes the comparison an involved task. Inspired by Tufte's small multiples [32], we have tried to make this comparison as straightforward as possible for the reader.

Figure 7 is built up in the following way:

- Each variable of interest is represented by a row of plots, which share the same $y$ axis. The value shown is the relative difference between the value of the respective variable obtained for a chosen number of representative days (indicated as title of the column), and the value from the full year optimisation;

Table 1: Description of design parameters used in Section 4.1 and their possible values

\begin{tabular}{llcc}
\hline Short name & Description & Unit & Possible values \\
\hline STC & STC STES volume & $\mathrm{m}^{3}$ & $\{50,100,150\} \times 10^{3}$ \\
WatGar & Storage volume Waterschei & $\mathrm{m}^{3}$ & $\{50,75,100,125\} \times 10^{3}$ \\
SolAr & STC area & $\mathrm{m}^{2}$ & $\{25,50,75,100\} \times 10^{3}$ \\
\hline
\end{tabular}




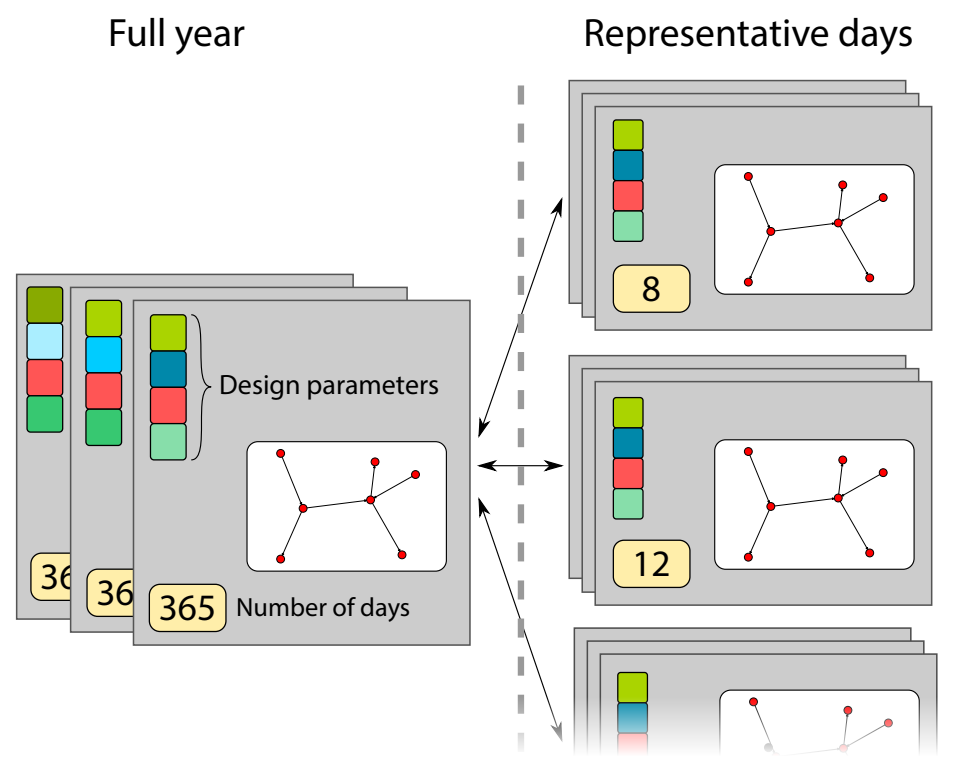

Figure 6: Schematic presentation to clarify the comparison of different deterministic designs for different representative days selections. Each card on the stacks represents a single modesto optimisation with the indicated number of days. Only cards with the same design (indicated by the small coloured squares) are compared here. A square with a different colour denotes a different value for that design parameter.

- Each selection of a number of representative days is represented in its own column of plots;

- Each plot has the same $x$ axis, namely the area of the STC field;

- Each dot represents a design choice with the STC area as indicated by the $x$ variable, and its colour shows the sum of the volumes of the Waterschei and STC storage tanks. The darker the dot, the more storage was installed; the influence of the position of the storage tanks in the network is not considered here.

- Dots may be shifted slightly to the left or the right for visibility, but this has no further meaning.

The objective functions of interest, namely the total electric energy use of the network pumps and backup heat pump and the total annualised costs 


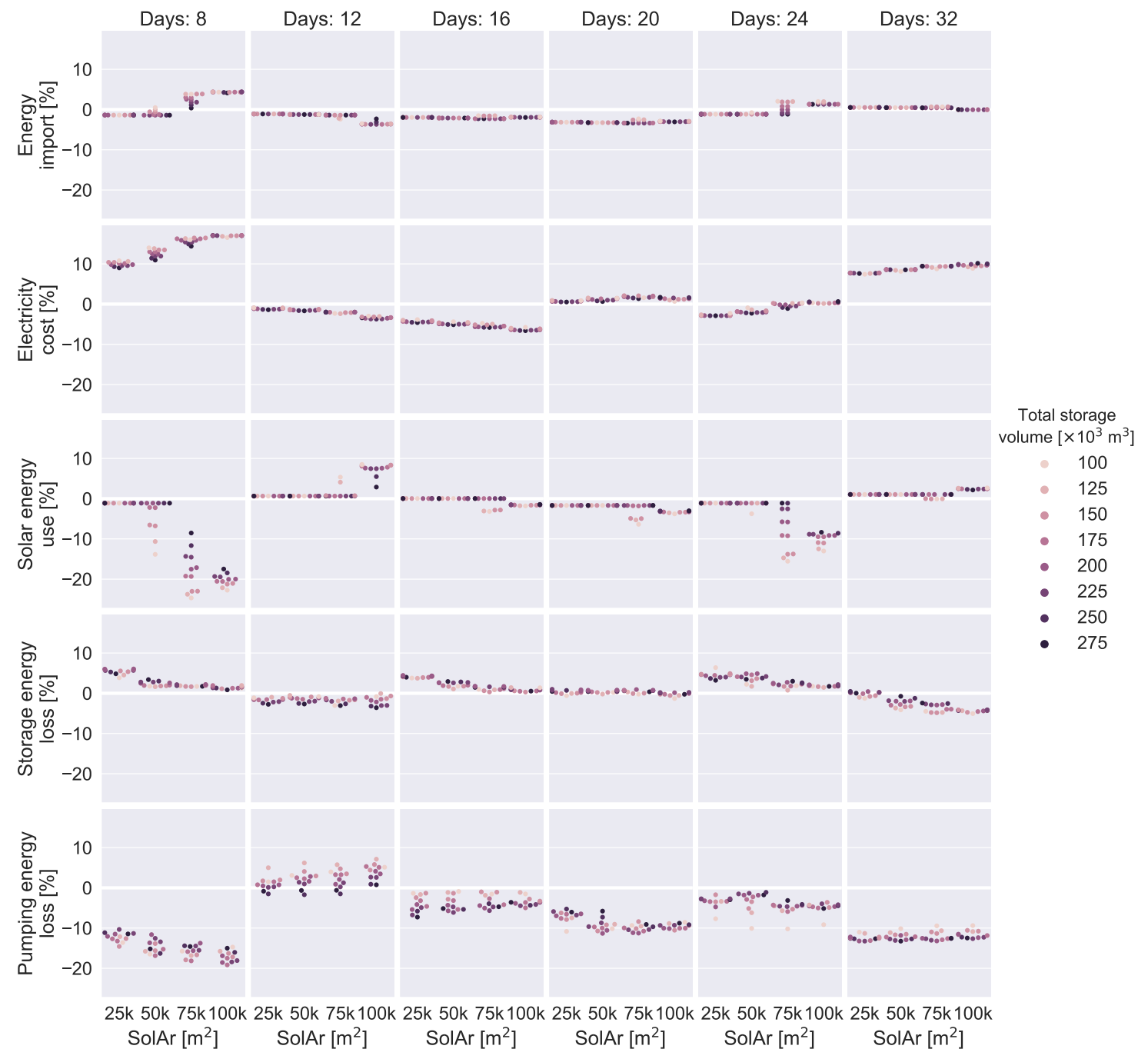

Figure 7: Comparison of the optimisation results for different representations of the studied designs. Percentage values indicate the relative difference from the full year result. 
are well approximated by most of the representative days selections. For the best selections, the error between the objective function values remains within $\pm 5 \%$ in the cost measure, which is a satisfying accuracy. For the backup energy use, the spread of the error is even smaller. The differences in backup energy use are complemented by those of the solar energy use, which is a logical result. The selections of 8 and 24 days seem most affected by differences in solar heat curtailment. Based on the deviations in the two objective functions, the selections of 12 and 20 days to represent the year seem the best options. The selection of 32 days is next in line but has a worse representation of the electricity costs.

The curtailment error (or larger absolute value deviations of the solar energy use) seems to be larger for systems with less storage volume. This appears logical because a smaller storage volume makes curtailment more likely to happen. Errors on curtailment are caused by differences in the SoC profile of the storage units. This is an inherent flaw of the methodology, given that the presented methodology requires the same charge/discharge profiles to be repeated on the same representative days. This requirement reduces the flexibility of the storage tanks to accomodate all of the solar production peaks, increasing the amount of curtailed heat.

Overall, the deviation is largest for the selection of 8 representative days. It was expected that the representation with the largest number of representative days, thus 32, would lead to the smallest error, but this is clearly not the case for storage losses and electricity cost.

Interestingly, whereas the differenc ${ }^{15}$ of backup energy use (and thus solar energy use) and electricity cost (neglecting the 8 days set) are hardly influenced by the design, the storage loss difference is much more sensitive to the design parameters. All selections display a decreasing deviation between the full optimisation and representative with increasing STC area. Some selections have a consistent influence of the storage volume ${ }^{16}$, but others don't have a clear pattern. Realising that the storage model has an exact representation of the storage losses as a function of the SoC, the difference in heat storage losses must result from a different average SoC during the year. This is a logical result of the requirement of equal charging/discharging

\footnotetext{
${ }^{15}$ Please note that this graph does not show the influence of the design variables on the optimisation outcome, but only on the difference between the representative and full optimisation.

${ }^{16}$ e.g., 12 days: smaller volume means relative difference closer to 0.
} 
patterns for the same representative days.

What is striking is that the relative differences for pumping energy are larger than the deviations of all other variables. This means that the representative days method is less suited to represent the model used for the pressure losses in the network. In this case, the 12 and 16 day selections show the smallest deviations, while others are closer to the $10 \%$ deviation line. Note that the pumping energy is small compared to the backup energy use, so the pumping losses should be of less concern.

\subsubsection{Calculation time reduction}

The aim of using representative days is of course to reduce the time needed to complete one OCP without losing too much accuracy.

These calculations were performed on a Dell Precision 7920 Tower with two Intel Xeon Silver 4116 processors $(2.1 \mathrm{GHz}-3.0 \mathrm{GHz}$ Turbo, 12 cores) and $64 \mathrm{~GB} 2666 \mathrm{MHz}$ DDR4 RAM. Thanks to an implementation using mutable parameters within the OCP formulation, the problem only needs to be compiled once for a given network and component structure 17 For the results presented so far, only a single thread was used. For the genetic algorithms, multithreading is employed to unlock the full potential of the workstation.

Judging from Figure 8a, a substantial calculation time reduction is clearly achieved. Given the large difference between the calculation time for the full optimisation and those using representative days, the different representative cases have been plotted in Figure $8 \mathrm{~b}$ for better comparison.

Looking at the solution time only (i.e., the point clouds in the lower half of the graphs), Figure $8 \mathrm{~b}$ suggests there is a close to linear relationship between the number of representative days and the solution time. This is to be expected from the linear optimisation algorithm, where the number of state variables increases linearly with the number of time steps considered. The compilation time ${ }^{18}$ seems to be rather constant for the representative days cases, namely between 6 and $7 \mathrm{~s}$. For the full optimisation, the compilation time is close to $35 \mathrm{~s}$, or in comparison more than half of the calculation

\footnotetext{
${ }^{17}$ The compilation time is defined as the time spent by modesto and Pyomo to transform the optimisation problem definition into a solver-readable format. This compilation time scales with the number of time steps considered, as well as the number of variables and equations.

${ }^{18}$ The difference between the top outlier and the average of the solution times cloud for each of the number of optimised days.
} 


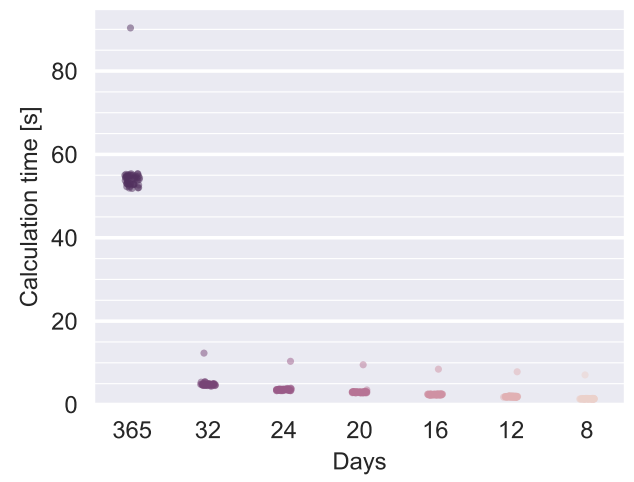

(a)

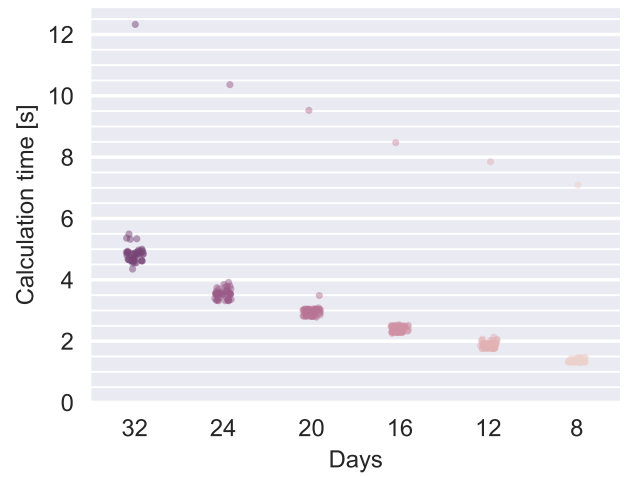

(b)

Figure 8: Comparison of calculation times for full year optimisation and different representative day selections. The outlier on the top always shows the sum of compilation and solution time, whereas the point cloud shows the distribution of solver time only after the first compilation.

Note that Figure $8 \mathrm{~b}$ is a repetition of Figure $8 \mathrm{a}$, with the full year optimisation times removed for better readability of the difference in optimisation time for the representative day cases.

time. For the representative days case, the compilation time would even surpass the solution time by at least $100 \%$, fully justifying the choice to use recompilation thanks to the mutable optimisation parameters.

More importantly, Figure 8 shows a reduction of around $55 \mathrm{~s}$ for the full calculation to between $1.5 \mathrm{~s}$ for 8 days to about $5 \mathrm{~s}$ for 32 days. On average, this means a speed up of 11 to over 36 times of the solution time excluding compilation time.

\subsection{Comparison of design optimisation outcome for representative days}

To compare the results of the design optimisation for different sets of representative days and the full year representation, the reference case described in Section 3 is subject to an evolutionary design optimisation with decision variables as indicated in Table 2 .

The optimisation produces 80 generations (excluding the initial population) of 48 individuals. Whereas in Section 4.1, we could still compare optimisations with the same design parameters, for the design optimisation this is no longer possible. Since the genetic algorithm is based on a randomised evolution, it is not possible to directly compare the solutions from different selections of representative days. 
Table 2: Summary of decision variables used for the genetic algorithm.

\begin{tabular}{llccc}
\hline Short name & Description & Unit & Lower bound & Upper bound \\
\hline Prod & Buffer tank near central heat pump & $\mathrm{m}^{3}$ & 0 & 3000 \\
ProdPow & Central heat pump nominal power & $\mathrm{MW}$ & 1 & 150 \\
STC & Storage tank near STC & $\mathrm{m}^{3}$ & 0 & $300 \times 10^{3}$ \\
SolAr & STC area & $\mathrm{m}^{2}$ & 0 & $400 \times 10^{3}$ \\
TerWes & Storage tank in Termien West & $\mathrm{m}^{3}$ & 0 & $200 \times 10^{3}$ \\
WatGar & Storage tank in Waterschei Garden & $\mathrm{m}^{3}$ & 0 & $200 \times 10^{3}$ \\
\hline
\end{tabular}

\subsubsection{Comparing the outcome of the different evolutions}

A good starting point is to compare the objective values. The backup energy use of the central heat pump and the total annualised costs are chosen as the two objectives. Figure 9 shows the fronts of non-dominated ${ }^{19}$ solutions for the full optimisation and for each of the representative days selections.

All non-dominated solution fronts largely follow the same trajectory, also with rather similar end points in the low cost, high energy import solution region. For the high investment, low energy import solutions, there is a larger spread on the location of the end points of the pareto fronts. The selections with 16, 20 or 24 representative days resemble the full optimisation solution front best, whereas the selection with 8,12 and surprisingly 32 days clearly have a larger deviation.

A common measure for the convergence of a genetic algorithm is the hypervolume of the pareto-efficient solutions (see [33]). In the case of an optimisation with two objectives, this measure is the area enclosed by the non-dominated solutions and a fixed reference point. With improving solution quality, the hypervolume increases, until no better solutions are found and the hypervolume stagnates.

The evolution of the hypervolume indicator is plotted in Figure 10 for the different selections of representative days along with the full optimisation. For the sake of comparison, a fixed number of generations has been calculated, and there was no stopping criterium based on convergence. Obviously, the evolutions are not the same, but at least they have a similar order of magnitude and trend. All trajectories seem to have almost reached convergence after around 40 generations of the optimisation algorithm, staying

\footnotetext{
${ }^{19}$ Non-dominated means there are no solutions that perform better on all objectives simultaneously.
} 


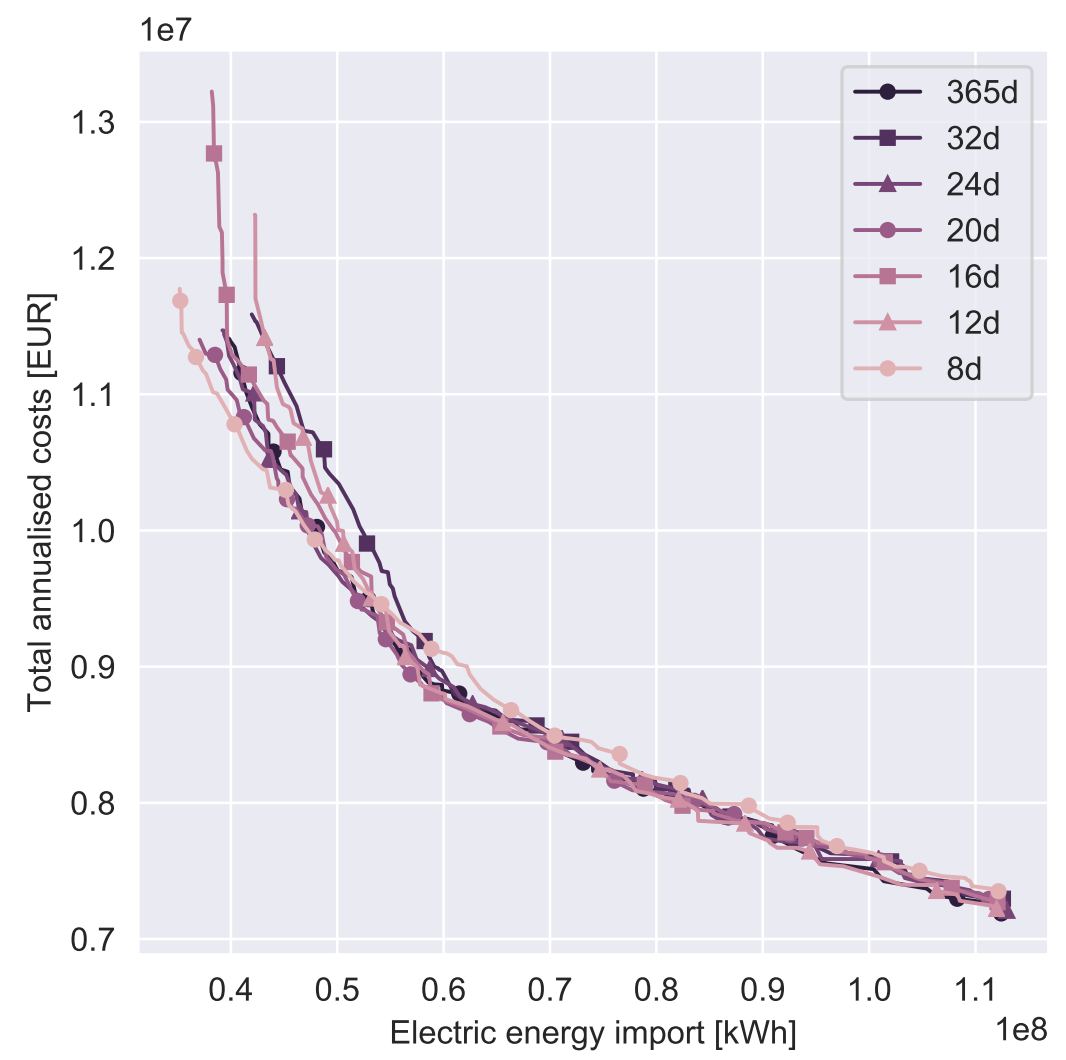

Figure 9: Non-dominated solutions for every representative days selection. A solid line connects all solutions that are pareto-efficient. The figure shows a good agreement between the different Pareto fronts, although towards the higher cost range discrepancies become clearer.

around the same hypervolume value until the $60^{\text {th }}$ generation.

Given the free evolution between the design limits and the large number of variables, it is challenging to assess whether the solution sets are similar for the different representative days selections. One technique that gives a more qualitative idea of the similarity of the non-dominated solutions is the parallel coordinates plot. Every individual from the non-dominated solution 


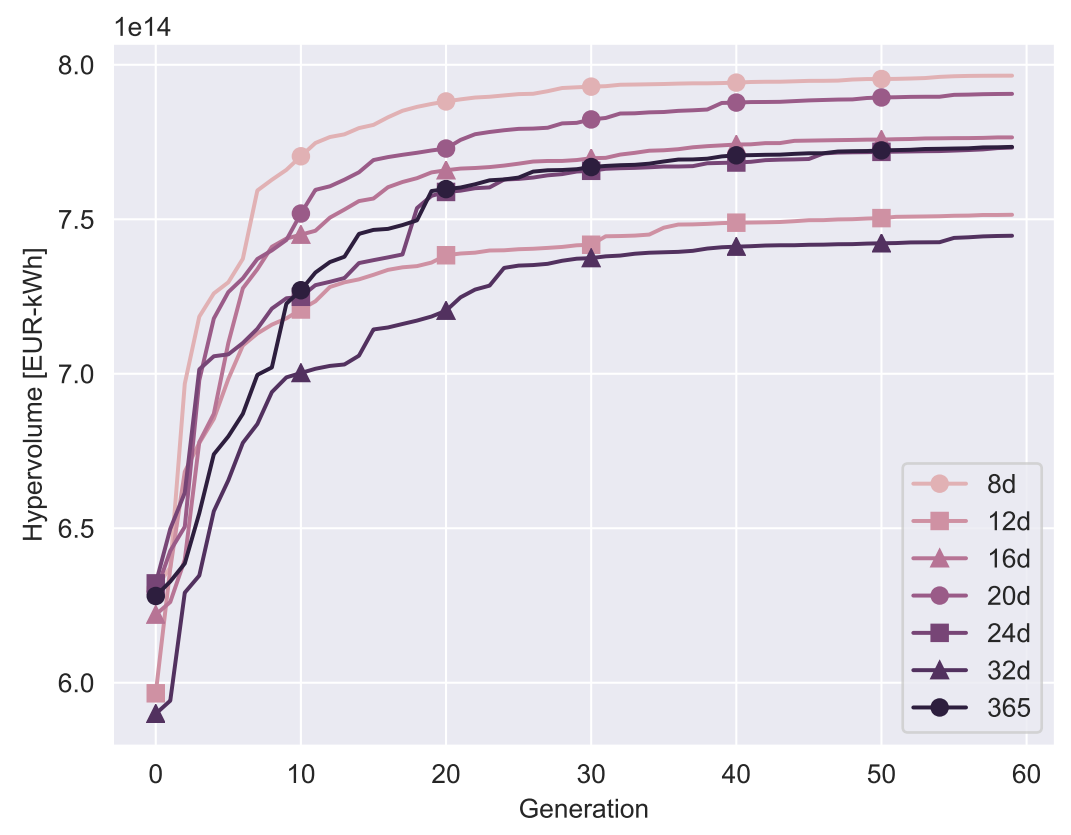

Figure 10: Hypervolume evolution for all representations. All hypervolumes use the same reference point for the calculation. All cases display the same convergence behaviour, but the final value of the different hypervolume indicators differs to a substantial degree. 
set is represented by a line in Figure 11. This line connects the normalised ${ }^{20}$ values of all design variables together with the two objective function values. The plot is split up according to the number of days that was accounted for in the optimisation. The normalisation of the values is performed based on the same minimum and maximum values for all selections of representative days.

Since the lines in Figure 11 are partly transparent, areas where a lot of lines meet will be darker, pointing at a similarity between part of the optimal solutions. There is an emphasis towards a lower storage volume for the TES tank at the production site, and the maximum heat pump power is clearly in the lower range. Either a high STC storage volume is chosen, or a low one, with only few solutions in between, and the same is true for the other storage tanks (TerWes and WatGar). As seen before in the pareto front, the costs are evenly spread, as are the annual energy use values, with a very clear inverse relation between the two.

Another clear trend focuses on higher STC areas, nearly always with the maximum allowed STC storage volume. This type of solution usually comes with large storage volume in the other locations as well, although there are a few 'crossovers' where one of the neighbourhood storage tanks is large and the other smaller. These solutions end at the higher end of the cost scale, but with a lower annual energy import from outside the district.

The parallel coordinates plot also allows getting an idea of the distribution of different design parameters, and how these distributions vary across different representations. All representations show a large variety in the installed STC area, and most have a focus of the heat pump size towards the lower end of the range. Most representations also suggest that the storage tanks are made either very large or very small, leaving a gap in between. However, the STC and WatGar STES systems are on average larger than the TerWes one. The smaller buffer tank near the heat pump has a wider spread, but usually the emphasis is towards the lower end of the range. Interestingly, most representations show similar storage 'crossovers' as the full optimisation, although the direction differs.

\footnotetext{
${ }^{20}$ The design values are normalised with respect to their limiting values (see Table 2), the objective values are normalised with respect to their respective extreme values across all representative days selections.
} 


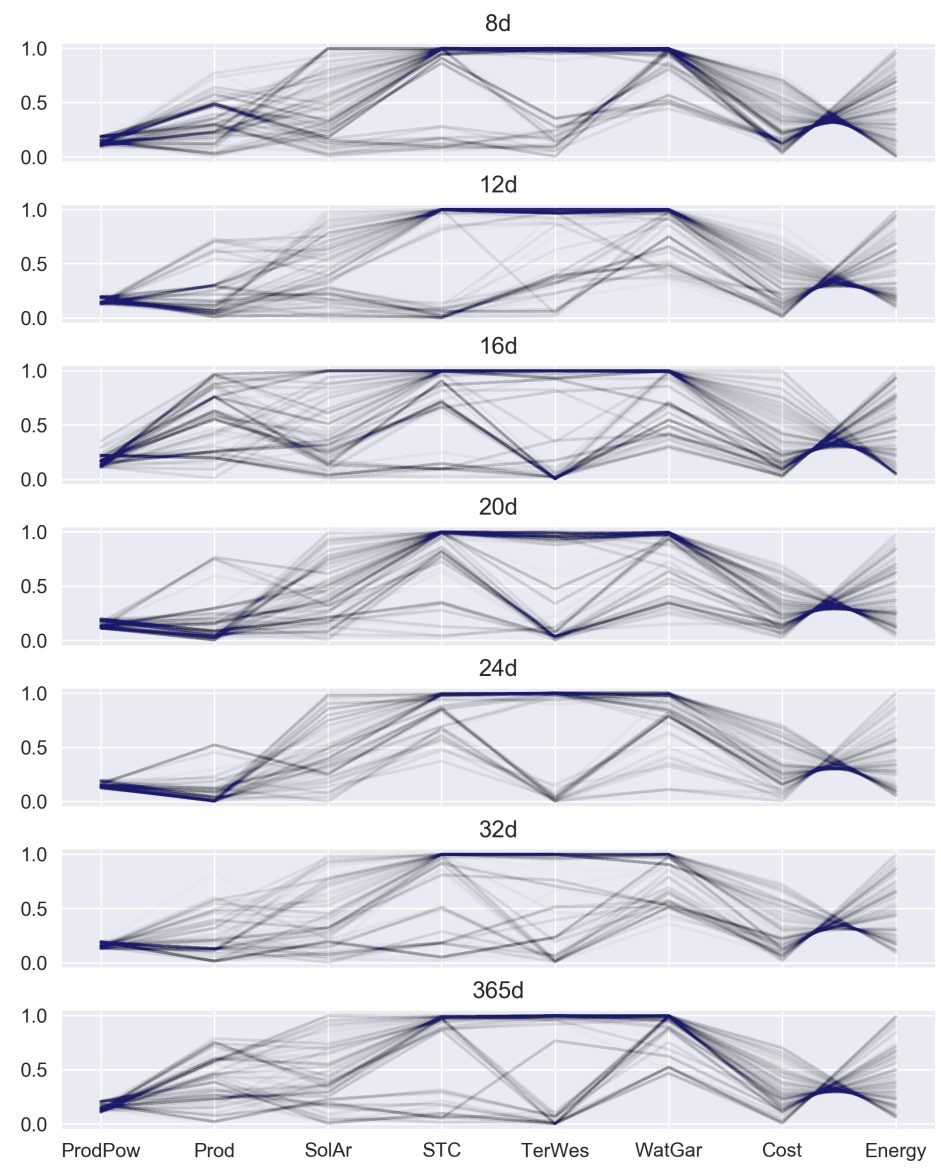

Figure 11: Parallel coordinates plot of non-dominated solutions, grouped per representation. Darker areas indicate a larger concentration of solutions for that parameter. All cases show a rather clear distinction between solutions with either a large installed STES volume, or a rather low one. On the other hand, the solar collector areas are evenly distributed over the available design choices. Overall, the similarity between the parallel coordinates plots is clear. 


\subsubsection{Calculation time reduction}

The calculation time reduction is not straightforward to compare across single individuals in all of the representative days selections. The reason is that the solution time also depends on how 'easily' a certain design can be optimised. An infeasible solution is detected quite quickly, but some feasible solutions have been observed to take considerable longer before the linear optimisation problem is solved.

However, comparing the full evolution of all of the compared representative days selections, it is clear that the full year optimisations take the longest time to complete (up to 4 days for 60 generations of 48 individuals, although some memory issues and errors prevented from exactly measuring the required time), whereas the representative cases only take a few hours to complete. The other calculation times are summed up in Table 3 . The evolution of the calculation times seems to suggest a superlinear evolution, which might be explained by the increasing overhead of data reading and writing.

Table 3: Calculation times of genetic algorithm for different representative days selections.

\begin{tabular}{lr}
\hline Nr. of days & Full solution time $[\mathrm{s}]$ \\
\hline 8 & 3162.3 \\
12 & 6616.1 \\
16 & 14324.5 \\
20 & 19992.4 \\
24 & 28203.5 \\
32 & 44370.5 \\
\hline
\end{tabular}

\section{Discussion}

We have shown good accordance between the operational and design optimisation results with representative days and the respective full year optimisations. This shows only one case, and the validity for other types of energy systems and other configurations should be checked as well. Still, there are no clear signs that indicate that the use of representative days would produce less accurate results for other design cases.

Furthermore, the representative day selection optimisation could perform differently with different numbers of input time profiles, or different types of data. Weather data for different climates could also have an impact on the 
quality of the selection. Both cases where the selection becomes better (less short-term variability, better correlation between the profiles) or worse (lack of correlation) could be imagined.

Comparing to similar studies, similar speed-ups with representative days are found. Kotzur et al. [16] reported a calculation time reduction from $109.2 \mathrm{~s}$ for the full year optimisation (MILP including design optimisation, for a smaller reference case) to only $6.0 \mathrm{~s}$ for the 12 representative days case. This means a speed-up by a factor 18.2, which is similar to the ca. 27 times speed-up found in our operational optimisation validation. Note that our validation did not consider design variables or integers, which complicates a direct comparison. In addition, Kotzur et al. studied an optimisation with a single objective, whereas we opted for a multi-objective design optimisation. Note that the studied systems are also quite different regarding their composition of conversion, transport and storage components. Furthermore, our models considered a higher level of detail. We have not been able to compare the difference between the k-medoids clustering approach Kotzur et al. used and our own optimal representative day selection and reordering algorithm in terms of accuracy.

Gabrielli et al. 9] reported a speed-up of at least 24 times (from $23 \mathrm{~h}$ for the full year case to maximum $1 \mathrm{~h}$ for 72 representative days). This is clearly a bigger speed-up than in the system considered here. However, the spatial complexity of the reference system studied here is larger, and for our case it was not possible to formulate the problem as a MILP.

A final discussion point is the fact that we only presented a limited variation of the number of days in the representative day set. A more exhaustive check of different numbers of days could identify a clear optimum for the selection, whereas now such a conclusion is not yet possible.

\section{Conclusion}

This paper presented a cross-comparison of a genetic design optimisation algorithm for a solar district heating system with seasonal thermal energy storage, using different numbers of representative days. An additional optimisation problem selected sets of representative days, and thereafter the ordering of the representative days to reorder the set into a full year was

optimised as well. This step made sure chronology was conserved, which is crucial for seasonal storage models. 
The cross-comparison was performed on two levels for the same reference optimisation case: firstly, the operational level was compared, to check the objective function values of the optimal energy flow problem for the same design combination with different numbers of representative days. This comparison showed that the representative day method is able to replicate the behaviour of the full year operational optimisation with acceptable accuracy, and with a 11 to 36 times faster calculation, depending on the number of representative days. This operational optimisation was used as the evaluation function for a genetic algorithm, which optimised the design parameters in the reference case. Comparing the resulting pareto-optimal results proved less conclusive due to the large dimensionality of the output data, however very similar outcome patterns have been found for all representative days selections. The calculation time reduction for the design level was even better than for the operational level. The long calculation times for the full year case furthermore made it very clear that the use of time aggregation methods is essential for more complex and realistic design problems, given the relatively small scale of the reference design case.

A possible future improvement is to perform a more exhaustive search of the number of days that is needed for a good approximation, as the current results don't clearly show the minimum required number of representative days for a certain level of accuracy. Furthermore, there was no clear trend of increasing accuracy for an increasing number of representative days. However, it was clear that the optimisations with only 8 representative days performed worse than the other selections. Like Kotzur et al. [16], our results led us to conclude that 12 representative days are enough to aptly approximate the full year optimisation results.

Finally, it is left as further work to apply the presented method to a larger and more realistic case study, presenting the outcome of the design optimisation in detail.

\section{Acknowledgement}

We want to thank Luca Scapino and Jan Diriken for the fruitful discussions that have contributed to this work. Furthermore, we would like to thank Jonas Timmerman for the useful feedback on the conference paper [17] that has been the starting point of this paper. Finally, we want to acknowledge the work of Ina De Jaeger to collect the data in the Genk building database 
and to transform it into model parameters we could use for the heat demand simulations.

The authors gratefully acknowledge EFRO/SALK for funding the research work of Bram van der Heijde and Annelies Vandermeulen through the project 'Towards a Sustainable Energy Supply in Cities', which received the support of the European Union, the European Regional Development Fund ERDF, Flanders Innovation \& Entrepreneurship and the Province of Limburg. The work of Bram van der Heijde and Annelies Vandermeulen is currently financed by VITO through a PhD Fellowship.

\section{Appendix A. Results of representative days selection}

Figures A.12 A.17present the comparison between the original input time series (blue) and the reconstructed profiles based on the different selections of representative days. Overall, the seasonally varying profiles are approximated very well. Due to faster variations, the solar profile and the electricity cost profile show a larger discrepancy with the original data, but still the recombination is not particularly bad.

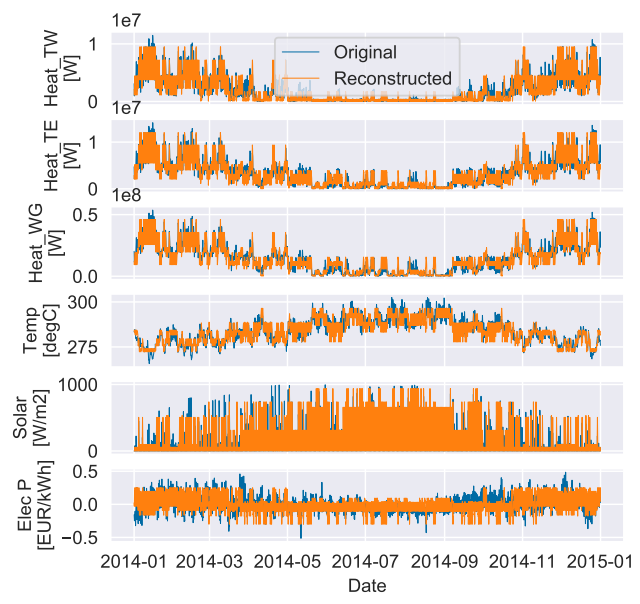

(a)

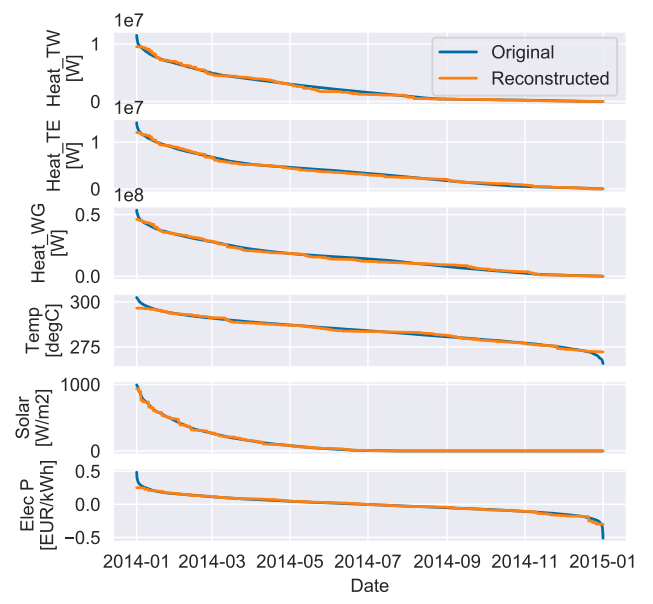

(b)

Figure A.12: Annual time series and DCs for the selection of 8 representative days.

\section{Appendix B. Cost data}

Table B.4 provides an overview of investment costs of the considered technologies, together with the annual fixed maintenance costs as a percentage 


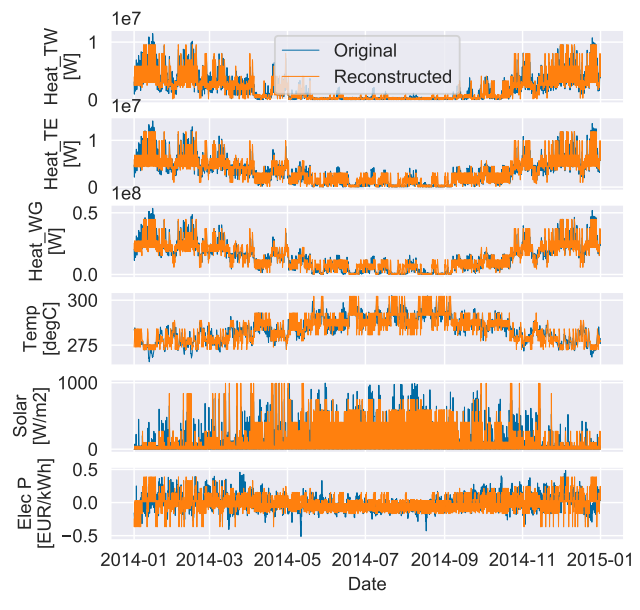

(a)

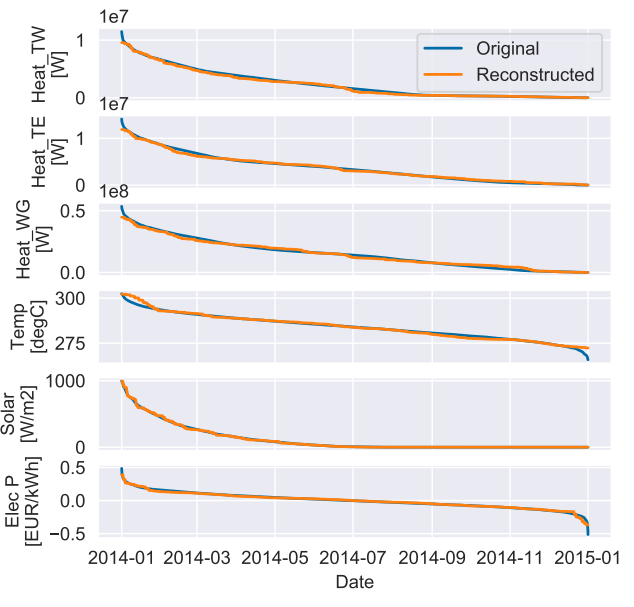

(b)

Figure A.13: Annual time series and DCs for the selection of 12 representative days.

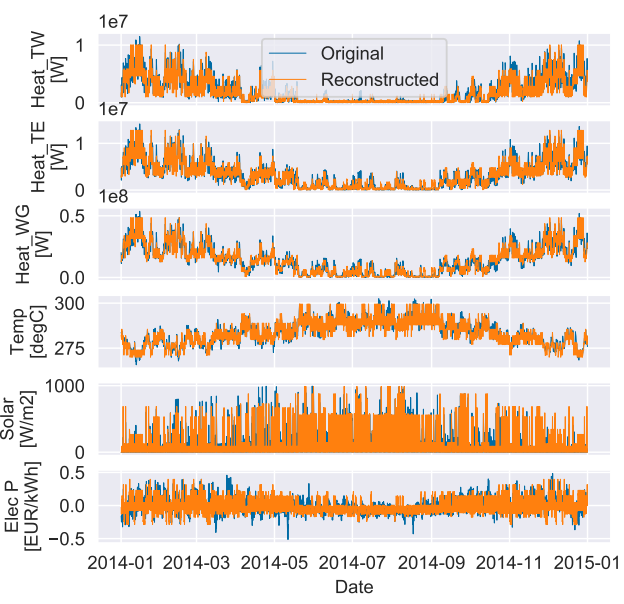

(a)

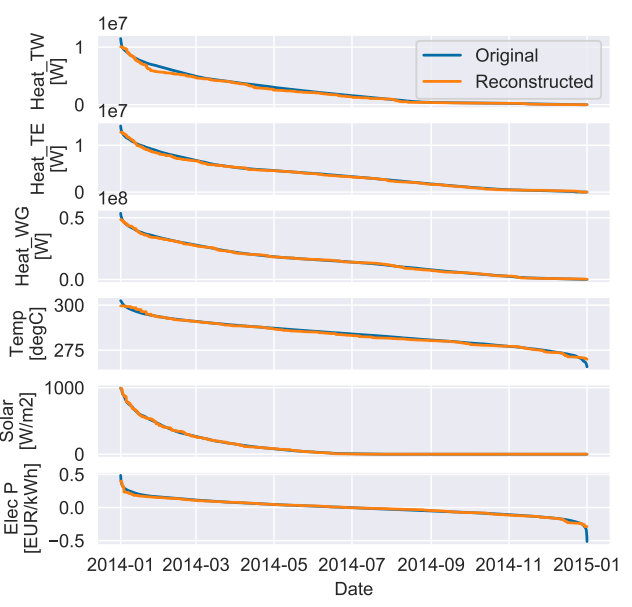

(b)

Figure A.14: Annual time series and DCs for the selection of 16 representative days. 


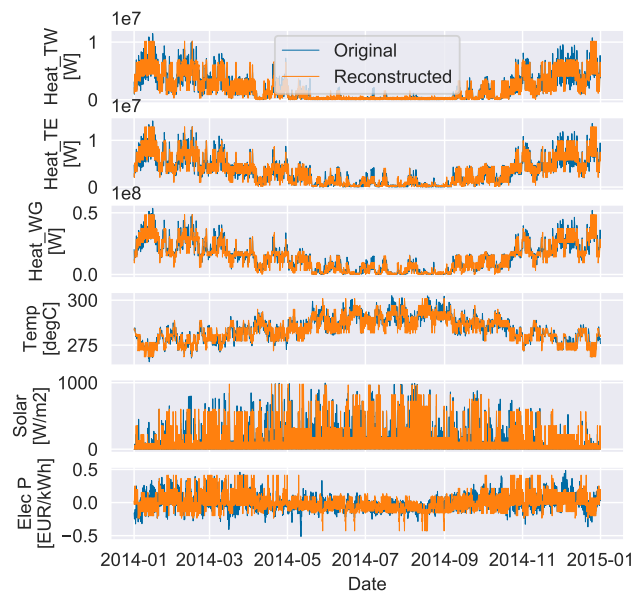

(a)

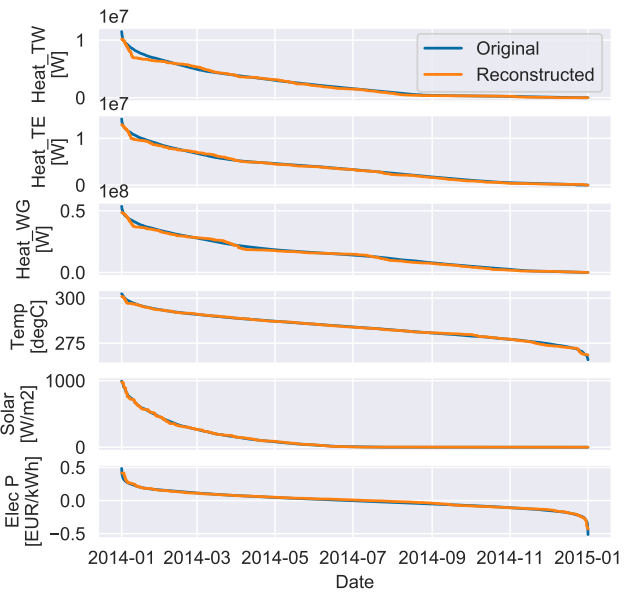

(b)

Figure A.15: Annual time series and DCs for the selection of 20 representative days.

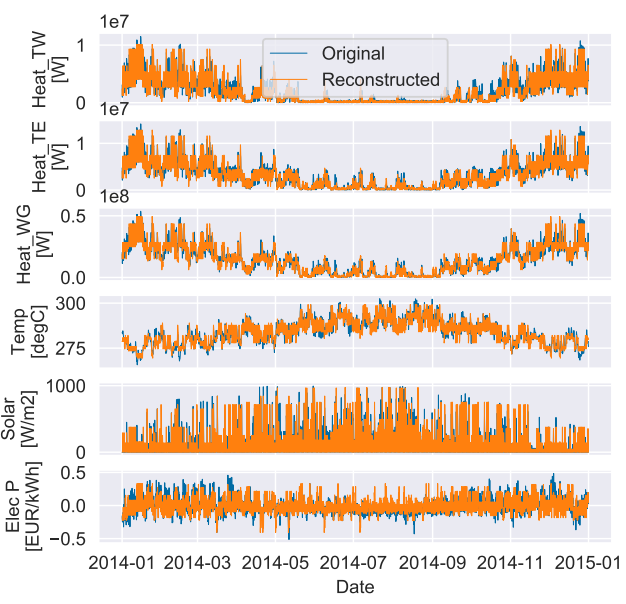

(a)

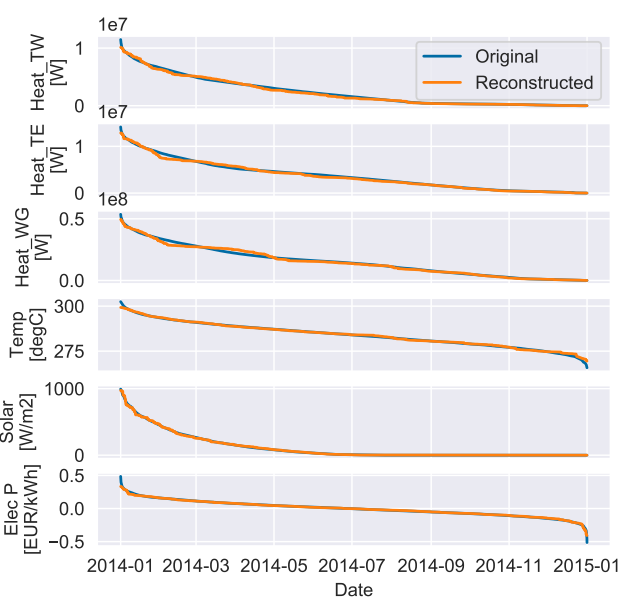

(b)

Figure A.16: Annual time series and DCs for the selection of 24 representative days. 


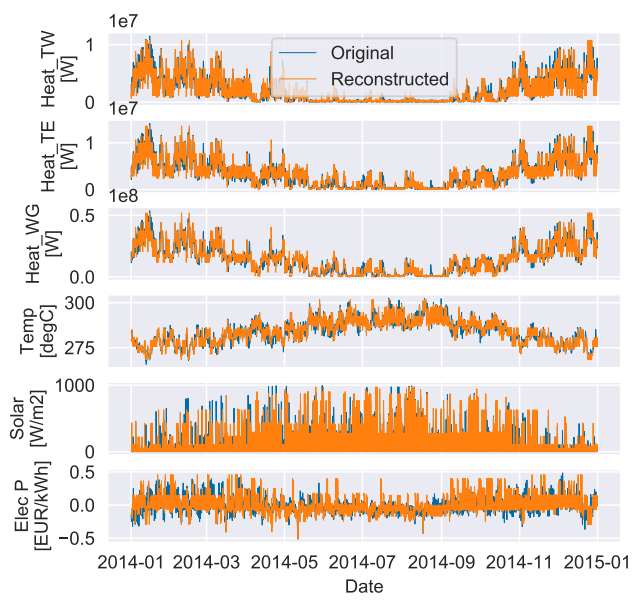

(a)

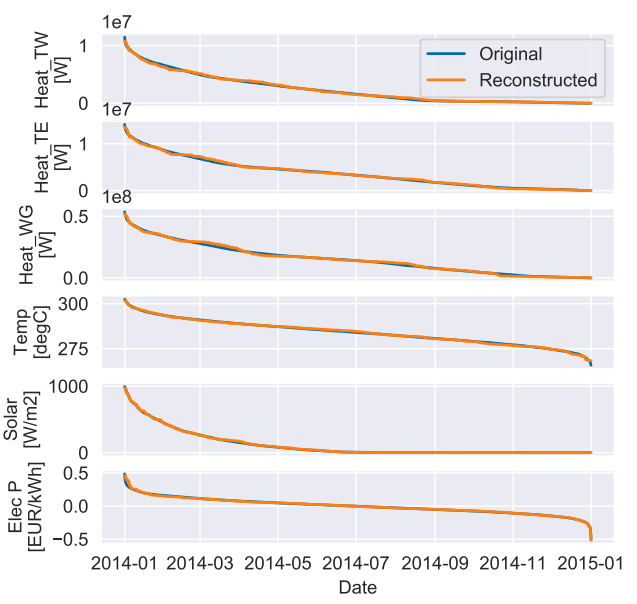

(b)

Figure A.17: Annual time series and DCs for the selection of 32 representative days.

of the total investment and the economic lifetime. The table also indicates the source of the data.

Table B.4: Summary of economic data used in the analysis.

\begin{tabular}{lrrrc}
\hline Technology & Investment cost & Fixed maintenance & Lifetime & Ref. \\
\hline Heat pump & $0.79 \mathrm{EUR} / \mathrm{W}$ & $0.60 \%$ & $20 \mathrm{y}$ & {$[34$} \\
STC & $250 \mathrm{EUR} / \mathrm{m}^{2}$ & $0.13 \%$ & $30 \mathrm{y}$ & {$[35$,} \\
TES systems & see Fig. B.18 & $0.70 \%$ & $20 \mathrm{y}$ & {$[29,[36$} \\
\hline
\end{tabular}

The cost of the heat pump is referenced to the heating power of the component, i.e. the power that is injected into the district heating system. The lifetimes are economic lifetimes, and they were taken from the cost databases of the Danish Energy Agency [34] and the EnergyPlan Modelling Team [29].

Since the unit cost of large storage systems varies with their size, the used cost data is represented in a graph in Figure B.18. 


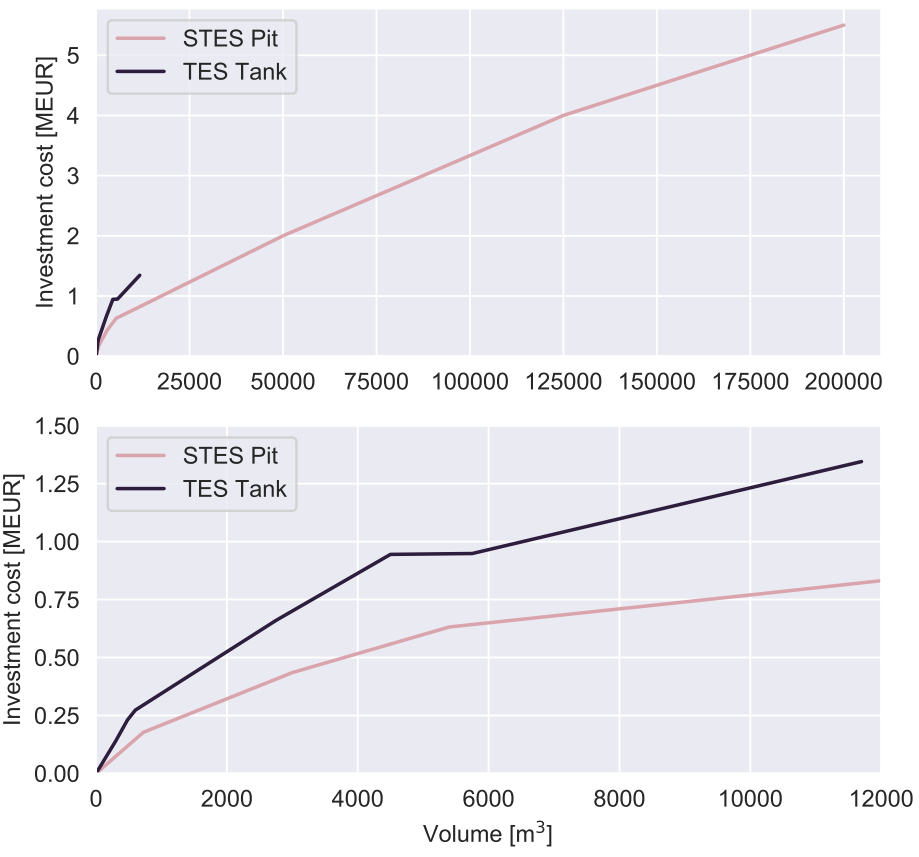

Figure B.18: Cost evolution in function of volume for TES tanks and STES pits, using a linear interpolation between known investment costs for real systems. Data derived from Schmidt and Miedaner 36.

Note that the lower graph is representing the same data as the upper plot, but focussing solely on the TES tank data. 


\section{Appendix C. Building heat demand simulations}

The heat demand is considered to be fixed and not influenced by the heat supply system. Therefore, the calculation of the deterministic heat demand is performed as a pre-processing step.

A CityGML database of building geometries in Genk is used (see De Jaeger et al. [37]). The CityGML information is translated into a set of building parameters for each building, including wall thicknesses, roof and wall areas, building materials etc. The building age is assumed to be fixed for the whole neighbourhood, and this influences the assumptions made about the insulation quality of the models.

The CityGML geometry data is translated to a resistance-capacitance (RC) model with an adapted version of the TEASER Python toolbox (Remmen et al. [38]) by De Jaeger et al. [37. The chosen model structure is the FourElement model, which is the most detailed model structure available in TEASER. The parameter values of thermal resistances and capacitances in the model are based on the physical building components. The thermal capacity of the air volume is multiplied by 5 to represent the thermal inertia of furniture in the building, as suggested by Sourbron [39].

The buildings are assumed to have an ideal heating system with a maximum nominal power rating based a steady-state design calculation for each building, considering an extremely cold day with an outdoor temperature of $-8^{\circ} \mathrm{C}$, a ground temperature of $10^{\circ} \mathrm{C}$ and an indoor temperature of $21^{\circ} \mathrm{C}$. The heating input to the building is calculated by an optimal control problem that makes sure the indoor temperatures are always within the comfort boundaries, which are determined using the StROBe Python toolbox (Baetens and Saelens [40]). The building models are simulated for one year and with a time step of $900 \mathrm{~s}$. This time step was necessary to guarantee numeric stability of the model, which is discretised using an explicit scheme (Euler discretisation). An initialisation period of one week is used.

\section{References}

[1] Henrik Lund, Poul Alberg Østergaard, David Connolly, Iva Ridjan, Brian Vad Mathiesen, Frede Hvelplund, Jakob Zinck Thellufsen, and Peter Sorknæs. Energy Storage and Smart Energy Systems. International Journal of Sustainable Energy Planning and Management, 11: 3-14, 2016. ISSN 22462929. doi: 10.5278/ijsepm.2016.11.2. 
[2] A. Vandermeulen, B. van der Heijde, D. Patteeuw, D. Vanhoudt, R. Salenbien, and L. Helsen. modesto - a Multi-Objective District Energy Systems Toolbox for Optimization. In 5th International Solar District Heating Conference, Graz, April 2018. URL https://lirias. kuleuven.be/retrieve/509049.

[3] Dave Olsthoorn, Fariborz Haghighat, and Parham A. Mirzaei. Integration of storage and renewable energy into district heating systems: A review of modelling and optimization. Solar Energy, 136:49-64, October 2016. ISSN 0038092X. doi: 10.1016/j.solener.2016.06.054. URL http://www.sciencedirect.com/science/article/pii/ S0038092X16302353http://linkinghub.elsevier.com/retrieve/ pii/S0038092X16302353https://linkinghub.elsevier.com/ retrieve/pii/S0038092X16302353.

[4] Jarmo Söderman and Frank Pettersson. Structural and operational optimisation of distributed energy systems. Applied Thermal Engineering, 26(13):1400-1408, September 2006. ISSN 13594311. doi: 10.1016/ j.applthermaleng.2005.05.034. URL http://linkinghub.elsevier. com/retrieve/pii/S1359431105002164.

[5] Céline Isabelle Weber. Multi-objective design and optimization of district energy systems including polygeneration energy conversion technologies. Doctoral thesis, Ecole Polytechnique Fédérale de Lausanne (EPFL), 2008. URL http://library.epfl.ch/theses/?nr $=4018 \% 5$ Cnhttp: // biblion.epfl.ch/EPFL/theses/2008/4018/4018_abs.pdf.

[6] Samira Fazlollahi, Gwenaelle Becker, Araz Ashouri, and François Maréchal. Multi-objective, multi-period optimization of district energy systems: IV - A case study. Energy, 84:365-381, May 2015. ISSN 03605442. doi: 10.1016/j.energy.2015.03.003. URL http://linkinghub.elsevier.com/retrieve/pii/ S0360544215002856https://linkinghub.elsevier.com/retrieve/ pii/S0360544215002856.

[7] Boran Morvaj, Ralph Evins, and Jan Carmeliet. Optimising urban energy systems: Simultaneous system sizing, operation and district heating network layout. Energy, 116:619-636, December 2016. ISSN 03605442. doi: 10.1016/j.energy.2016.09.139. 
URL http://www.sciencedirect.com.kuleuven.ezproxy. kuleuven.be/science/article/pii/S0360544216314207https: //linkinghub.elsevier.com/retrieve/pii/S0360544216314207.

[8] Tobias Falke, Stefan Krengel, Ann-Kathrin Meinerzhagen, and Armin Schnettler. Multi-objective optimization and simulation model for the design of distributed energy systems. Applied Energy, 184:15081516, December 2016. ISSN 03062619. doi: 10.1016/j.apenergy. 2016.03.044. URL https://linkinghub.elsevier.com/retrieve/ pii/S0306261916303646.

[9] P. Gabrielli, M. Gazzani, E. Martelli, and M. Mazzotti. Optimal design of multi-energy systems with seasonal storage. Applied Energy, 219(May 2017):408-424, 2018. ISSN 03062619. doi: 10.1016/j.apenergy.2017.07. 142. URL https://doi.org/10.1016/j.apenergy.2017.07.142.

[10] Matteo Giacomo Prina, Matteo Lionetti, Giampaolo Manzolini, Wolfram Sparber, and David Moser. Transition pathways optimization methodology through EnergyPLAN software for long-term energy planning. Applied Energy, 235:356-368, February 2019. ISSN 03062619. doi: 10.1016/J.APENERGY.2018.10.099. URL https://www . sciencedirect.com/science/article/pii/S0306261918316672

[11] P. Nahmmacher, E. Schmid, L. Hirth, and B. Knopf. Carpe diem: A novel approach to select representative days for long-term power system modeling. Energy, 112:430-442, 2016. ISSN 03605442. doi: 10.1016/j.energy.2016.06.081. URL http://dx.doi.org/10.1016/j. energy.2016.06.081.

[12] K. Poncelet, H. Höschle, E. Delarue, A. Virag, and W. D'haeseleer. Selecting Representative Days for Capturing the Implications of Integrating Intermittent Renewables in Generation Expansion Planning Problems. IEEE Transactions on Power Systems, 32(3):1936-1948, 2017.

[13] D. Patteeuw and L. Helsen. Combined design and control optimization of residential heating systems in a smart-grid context. Energy and Buildings, 133:640-657, 2016. ISSN 03787788. doi: 10.1016/j.enbuild.2016. 09.030. URL http://dx.doi.org/10.1016/j .enbuild.2016.09.030. 
[14] J. Timmerman, M. Hennen, A. Bardow, P. Lodewijks, L. Vandevelde, and G. Van Eetvelde. Towards low carbon business park energy systems: A holistic techno-economic optimisation model. Energy, 125:747-770, April 2017. ISSN 03605442. doi: 10.1016/j.energy. 2017.02.081. URL http://linkinghub.elsevier.com/retrieve/pii/ S0360544217302554.

[15] M. Welsch, M. Howells, M. Bazilian, J. F. DeCarolis, S. Hermann, and H. H. Rogner. Modelling elements of Smart Grids - Enhancing the OSeMOSYS (Open Source Energy Modelling System) code. Energy, 46 (1):337-350, 2012. ISSN 03605442. doi: 10.1016/j.energy.2012.08.017. URL http://dx.doi.org/10.1016/j .energy .2012.08.017.

[16] L. Kotzur, P. Markewitz, M. Robinius, and D. Stolten. Time series aggregation for energy system design: Modeling seasonal storage. Applied Energy, 213(October 2017):123-135, 2018. ISSN 03062619. doi: 10.1016/j.apenergy.2018.01.023. URL https://doi.org/10.1016/j. apenergy.2018.01.023.

[17] B. van der Heijde, L. Scapino, A. Vandermeulen, D. Patteeuw, R. Salenbien, and L. Helsen. Using representative time slices for optimization of thermal energy storage systems in low- temperature district heating systems. In Jose Teixeira, editor, 31st International Conference on Efficiency, Cost, Optimization, Simulation and Environmental Impact of Energy Systems, Guimarães, Portugal, 2018. University of Minho.

[18] Renaldi Renaldi and Daniel Friedrich. Multiple time grids in operational optimisation of energy systems with short- and long-term thermal energy storage. Energy, 133:784-795, August 2017. ISSN 03605442. doi: 10.1016/j.energy.2017.05.120. URL http://dx.doi.org/10.1016/j.energy.2017.05.120https:// linkinghub.elsevier.com/retrieve/pii/S0360544217308782.

[19] Xenofon Floros, Federico Bergero, Nicola Ceriani, Francesco Casella, Ernesto Kofman, and François Cellier. Simulation of Smart-Grid Models using Quantization-Based Integration Methods. In Proceedings of the 10th International Modelica Conference, March 10-12, 2014, Lund, Sweden, volume 96, pages 787-797, March 2014. doi: 10.3384/ ecp14096787. URL http://www.ep.liu.se/ecp_article/index.en. aspx? issue $=96$; article $=82$. 
[20] William E Hart, Carl D Laird, Jean-Paul Watson, David L Woodruff, Gabriel A Hackebeil, Bethany L Nicholson, and John D Siirola. Pyomo - optimization modeling in python, volume 67. Springer Science \& Business Media, second edition, 2017.

[21] European Committee for Standardization (CEN). Thermal solar systems and components - Solar collectors - Part 2: Test methods, 2006.

[22] Institut für Solartechnik. SPF Online Kollektorkatalog, 2017. ISSN 0028-4793. URL http://www . spf . ch/index .php?id=111\&no_cache=1.

[23] Bram van der Heijde, Arnout Aertgeerts, and Lieve Helsen. Modelling steady-state thermal behaviour of double thermal network pipes. International Journal of Thermal Sciences, 117:316-327, July 2017. ISSN 12900729. doi: 10.1016/j.ijthermalsci.2017.03.026. URL http://dx.doi.org/10.1016/j.ijthermalsci.2017.03.026http: //linkinghub.elsevier.com/retrieve/pii/S1290072916317355.

[24] J. Vandewalle and W. D'haeseleer. The impact of small scale cogeneration on the gas demand at distribution level. Energy Conversion and Management, 78:137-150, February 2014. ISSN 01968904. doi: 10.1016/j.enconman.2013.10.005. URL http://dx.doi.org/10.1016/j.enconman.2013.10.005http: //linkinghub.elsevier.com/retrieve/pii/S0196890413006250.

[25] David Steen, Michael Stadler, Gonçalo Cardoso, Markus Groissböck, Nicholas DeForest, and Chris Marnay. Modeling of thermal storage systems in MILP distributed energy resource models. Applied Energy, 137:782-792, 2015. ISSN 03062619. doi: 10.1016/j.apenergy.2014.07. 036. URL http://dx.doi.org/10.1016/j.apenergy.2014.07.036.

[26] Peter Sorknæs. Simulation method for a pit seasonal thermal energy storage system with a heat pump in a district heating system. Energy, 152:533-538, June 2018. ISSN 03605442. doi: 10.1016/j.energy.2018.03. 152. URL https://doi.org/10.1016/j.energy.2018.03.152http: //linkinghub.elsevier.com/retrieve/pii/S0360544218305619.

[27] Félix-Antoine Fortin, François-Michel De Rainville, Marc-André Gardner, Marc Parizeau, and Christian Gagné. DEAP : Evolutionary Algorithms Made Easy. Journal of Machine Learning Research, 13:2171- 
2175, 2012. ISSN 1533-7928. doi: 10.1.1.413.6512. URL http://jmlr. csail.mit.edu/papers/volume13/fortin12a/fortin12a.pdf

[28] K Deb, Samir Agrawal, Amrit Pratap, and T Meyarivan. A Fast Elitist Non-Dominated Sorting Genetic Algorithm for Multi-Objective Optimization: NSGA-II. In International Conference on Parallel Problem Solving From Nature, pages 849-858, Berlin, Heidelberg, 2000. Springer.

[29] EnergyPLAN Modelling Team. EnergyPLAN Cost Database. Technical report, Sustainable Energy Planning Research Group, Aalborg University, 2018. URL https://www.energyplan.eu/useful_resources/ costdatabase/.

[30] F. Jorissen, G. Reynders, R. Baetens, D. Picard, D. Saelens, and L. Helsen. Implementation and verification of the IDEAS building energy simulation library. Journal of Building Performance Simulation, 11(6):669-688, November 2018. ISSN 1940-1493. doi: 10.1080/19401493.2018.1428361. URL https://doi.org/10.1080/19401493.2018.1428361https://www. tandfonline.com/doi/full/10.1080/19401493.2018.1428361.

[31] Ina De Jaeger, Annelies Vandermeulen, Bram van der Heijde, Lieve Helsen, and Dirk Saelens. Aggregating occupant profiles for simulating the district heat demand. To be submitted to Journal of Building Performance Simulation, 2019.

[32] Edward Tufte. Envisioning Information. Graphics Press, Cheshire, CT, USA, 1990. ISBN 0-9613921-1-8.

[33] Carlos M. Fonseca, Luís Paquete, and Manuel López-Ibáñez. An improved dimension-sweep algorithm for the hypervolume indicator. In Proceedings of the 2006 Congress on Evolutionary Computation (CEC 2006), pages 1157-1163. IEEE Press, Piscataway, NJ, July 2006. doi: 10.1109/CEC.2006.1688440.

[34] Danish Energy Agency and ENERGINET. Technology Data for Energy Plants Generation of Electricity and District Heating. Technical Report 12-2018, Copenhagen, 2016.

[35] Per Alex Sørensen, Jan Erik Nielsen, Riccardo Battisti, Thomas Schmidt, and Daniel Trier. Solar district heating 
guidelines: Collection of fact sheets. (August), 2012. URL http://solar-district-heating.eu/Portals/0/Factsheets/ SDH-WP3-D31-D32_August2012.pdf.

[36] Thomas Schmidt and Oliver Miedaner. Solar district heating guidelines - Storage Components Fact Sheet. Technical report, 2012. URL http://solar-district-heating.eu/Portals/0/Factsheets/ SDH-WP3_FS-7-2_Storage_version3.pdf.

[37] Ina De Jaeger, Glenn Reynders, Yixiao Ma, and Dirk Saelens. Impact of building geometry description within district energy simulations. Energy, 158:1060-1069, September 2018. ISSN 03605442. doi: 10.1016/j.energy.2018.06.098. URL https://linkinghub.elsevier. com/retrieve/pii/S0360544218311630.

[38] Peter Remmen, Moritz Lauster, Michael Mans, Marcus Fuchs, Tanja Osterhage, and Dirk Müller. TEASER: an open tool for urban energy modelling of building stocks. Journal of Building Performance Simulation, 11(1):84-98, January 2018. ISSN 1940-1493. doi: 10.1080/19401493.2017.1283539. URL https://doi.org/10.1080/19401493.2017.1283539https://www . tandfonline.com/doi/full/10.1080/19401493.2017.1283539.

[39] Maarten Sourbron. Dynamic thermal behaviour of buildings with concrete core activation. $\mathrm{PhD}$ Thesis, $\mathrm{KU}$ Leuven, Belgium, 2012. ISBN 978-94-6018-572-4. URL https://lirias.kuleuven.be/ handle/123456789/396689.

[40] Ruben Baetens and Dirk Saelens. Modelling uncertainty in district energy simulations by stochastic residential occupant behaviour. Journal of Building Performance Simulation, 9(4):431-447, July 2016. ISSN 1940-1493. doi: 10.1080/19401493.2015.1070203. URL http://www . tandfonline.com/doi/full/10.1080/19401493.2015.1070203. 